\title{
Presynaptic Clathrin Levels Are a Limiting Factor for Synaptic Transmission
}

\author{
Francisco J. López-Murcia, ${ }^{1,2}$ (D) Stephen J. Royle, ${ }^{3}$ and ${ }^{\circledR}$ Artur Llobet ${ }^{1,2}$ \\ ${ }^{1}$ Laboratory of Neurobiology, Bellvitge Biomedical Research Institute (IDIBELL), and ${ }^{2}$ Department of Pathology and Experimental Therapeutics, Faculty of \\ Medicine, University of Barcelona, 08907 L'Hospitalet de Llobregat, Spain, and ${ }^{3}$ Division of Biomedical Cell Biology, Warwick Medical School, University of \\ Warwick, Coventry CV4 7AL, United Kingdom
}

To maintain communication, neurons must recycle their synaptic vesicles with high efficiency. This process places a huge burden on the clathrin-mediated endocytic machinery, but the consequences of this are poorly understood. We found that the amount of clathrin in a presynaptic terminal is not fixed. During stimulation, clathrin moves out of synapses as a function of stimulus strength and neurotransmitter release probability, which, together with membrane coat formation, transiently reduces the available pool of free clathrin triskelia. Correlative functional and morphological experiments in cholinergic autapses established by superior cervical ganglion neurons in culture show that presynaptic terminal function is compromised if clathrin levels fall by $20 \%$ after clathrin heavy chain knock down using RNAi. Synaptic transmission is depressed due to a reduction of cytoplasmic and readily releasable pools of vesicles. However, synaptic depression reverts after dialysis of exogenous clathrin, thus compensating RNAi-induced depletion. Lowering clathrin levels also reduces quantal size, which occurs concomitantly with a decrease in the size of synaptic vesicles. Large dense-core vesicles are unaffected by clathrin knock down. Together, our results show that clathrin levels are a dynamic property of presynaptic terminals that can influence short-term plasticity in a stimulus-dependent manner.

Key words: clathrin; endocytosis; presynaptic; short-term depression; synaptic vesicle; synaptic vesicle pools

\section{Introduction}

Since the original description of clathrin as the major structural component of coated vesicles, it is generally considered that cells contain a virtually limitless reservoir of this protein (Pearse, 1976). In most cells, clathrin accounts for $\sim 0.1-0.2 \%$ of the total protein content (Doxsey et al., 1987). This estimate is fivefold higher in neurons (Goud et al., 1985) and is higher still at synaptic terminals, where clathrin is enriched (Saheki and De Camilli, 2012). These elevated levels are required to maintain neuronal communication because clathrin-mediated endocytosis (CME) is the predominant mechanism used to recycle synaptic vesicles (Granseth et al., 2006). Nonetheless, whether clathrin levels can become a limiting factor to presynaptic terminal function is still an open question.

An estimate of the available clathrin pool (ACP), or number of unassembled triskelia available for coat formation in a prototyp-

\footnotetext{
Received Dec. 5, 2013; revised May 19, 2014; accepted May 22, 2014.

Author contributions: S.J.R. and A.L. designed research; F.J.L.-M., S.J.R., and A.L. performed research; F.J.L.-M. and A.L. analyzed data; S.J.R. and A.L. wrote the paper.

This work was supported by El Ministerio de Economía y Competitividad (MINECO Grant SAF 2012-36375 to A.L.) and Fundacio La Marató de TV3 (A.L.). F.J.L.-M. was the recipient of a MINECO predoctoral fellowship (Grant BES2010-032355). We thank the Electron Microscopy unit (Campus Casanova) of Centres Científics i Tecnològics of the University of Barcelona for their involvement and support and Rachel Jones for technical support.

The authors declare no competing financial interests.

Correspondence should be addressed to either of the following: Artur Llobet, Laboratory of Neurobiology, Bellvitge Biomedical Research Institute (IDIBELL), 08907 L'Hospitalet de Llobregat, Spain, E-mail: allobet@ub.edu; or Stephen Royle, Division of Biomedical Cell Biology, Warwick Medical School, University of Warwick, Coventry CV4 7AL, UK, E-mail:S.J.Royle@warwick.ac.uk.

DOI:10.1523/JNEUROSCI.5081-13.2014

Copyright $\odot 2014$ the authors $\quad 0270-6474 / 14 / 348618-12 \$ 15.00 / 0$
}

ical non-neuronal cell, can be drawn from previous work. Considering an average amount of $0.5-1 \times 10^{6}$ clathrin triskelia/cell (Doxsey et al., 1987) and a ratio of assembled to unassembled clathrin of $\sim 1$ (Goud et al., 1985), the size of the ACP would be $2.5-5 \times 10^{5}$ triskelia. Because clathrin-coated vesicles (CCVs) contain 36-60 triskelia (Cheng et al., 2007), a single cell could make hundreds of vesicles without significantly depleting the ACP. However, three observations suggest that the ACP can be limiting at neuronal synapses. First, presynaptic terminals can entirely release their readily release pool (RRP) within $1 \mathrm{~s}$ when stimulated at a high physiological frequency (i.e., $20 \mathrm{~Hz}$ ). The instant release of $\sim 2 \%$ of all synaptic vesicles present in the terminal (Rizzoli and Betz, 2005) and subsequent retrieval through CME operating with a time constant of $\sim 15$ s (Balaji and Ryan, 2007; Granseth and Lagnado, 2008) would transiently reduce the concentration of free triskelia. Second, clathrin moves out of presynaptic terminals during stimulation. For example, stimulation of hippocampal boutons with 400 action potentials (APs) at 20 Hz causes an $\sim 3 \%$ transient decrease in presynaptic clathrin concentration (Granseth et al., 2006). Third, the lifetime of clathrincoated intermediates is $\sim 1 \mathrm{~min}$ (Miller and Heuser, 1984). Therefore, for a $20 \mathrm{~Hz}$ stimulation, the ACP would constantly decrease for 1200 APs.

Although these observations suggest that clathrin levels decrease significantly during high-frequency stimulation, the possible link between the reduction in the ACP and synaptic depression has not yet been investigated. Previous work has shown that, in non-neuronal cells, clathrin concentration in the 
cell is related to CME by a Hill function with a cooperativity index $>6$, such that small changes in clathrin levels lead to a significant impairment of endocytosis (Moskowitz et al., 2005). This conclusion was reached by performing a correlative analysis of transferrin receptor endocytosis and clathrin levels after RNAi of clathrin heavy chain $(\mathrm{CHC})$ in a cell line. In the present study, we established a comparable experimental approach in a neuronal system: cholinergic autaptic single-cell microcultures (SCMs) (PerezGonzalez et al., 2008). We found that release of neurotransmitters is affected by decreases in clathrin levels that were as low as $\sim 20 \%$, showing the high sensitivity of synaptic transmission to clathrin availability.

\section{Materials and Methods}

Molecular biology. For expression of EGFP-tagged clathrin light chain (neuronal splice form), the coding sequence of GFP-nLCa was amplified by PCR to introduce MluI and SalI sites and was cloned into pWPT-GFP (plasmid 12255; Addgene), replacing GFP with GFP-nLCa. For CHC RNAi, pLVTHM vectors (plasmid 12247; Addgene) were used to coexpress shRNA and a GFP marker. Briefly, a ClaI-EcoRI fragment was exchanged with either pBrain-SpH-shGL2 (control) or pBrain-SpHshCHC1 (rat CHC). GFP-nLCa and pBrain vectors were available from previous work (Royle et al., 2005). For lentivirus production, HEK 293T cells were transfected by the calcium phosphate method following methods described by Didier Trono (http://tronolab.epfl.ch/lentivectors) with pMD2G (plasmid 12259; Addgene), pCMVR8.74 (plasmid 22036; Addgene), and the appropriate pLVTHM constructs. Two days later, culture medium containing lentiviral particles was collected in 3 rounds at $8 \mathrm{~h}$ intervals, kept at $4^{\circ} \mathrm{C}$, and centrifuged at $500 \times \mathrm{g}$. Supernatants were aliquoted and stored at $-80^{\circ} \mathrm{C}$.

Cell culture. Experimental procedures were approved by the Department of Environment from the Generalitat de Catalunya. SCMs from superior cervical ganglion neurons were prepared following the previously described methods using postnatal day 0 (P0) to P2 Sprague Dawley rats (Perez-Gonzalez et al., 2008; Albrecht et al., 2012). Briefly, medium containing all dissociated ganglionic cells was placed in a 100$\mathrm{mm}$-diameter culture dish for $90 \mathrm{~min}$ at $37^{\circ} \mathrm{C}$. At the end of this preplating period, $\geq 95 \%$ of non-neuronal cells were found to be adhered to the dish, but most neurons remained in suspension. Medium was then collected and neurons seeded at 2500 cells $\cdot \mathrm{ml}^{-1}$ on $15 \mathrm{~mm}$ coverslips containing 10-20 collagen microdrops of 100-400 $\mu \mathrm{m}$ diameter. Culture medium was DMEM/F12 [1:1] containing 2.5\% fetal bovine serum, $2.5 \%$ rat serum (prepared in the animal care facility of the Health Universitat de Barcelona campus), 3-5 nM NGF, 2 nM CNTF (Alomone Labs), and $25 \mathrm{U} / \mathrm{ml}$ penicillin/streptomycin at $37^{\circ} \mathrm{C}$ and $8 \% \mathrm{CO}_{2}$. To genetically modify SCMs, lentiviral infection was applied at $5 \mathrm{~d}$ in vitro (DIV) during a 1:2 overnight incubation of the appropriate viral stock. GFP fluorescence was evident $4-6 \mathrm{~d}$ after infection.

Electrophysiological recordings and imaging. All experiments were performed in the whole-cell configuration of patch-clamp mode using neurons microcultured for 16-20 DIV. Typical resistances of pipettes used for recordings were 3-5 $\mathrm{M} \Omega$ when filled with internal solution composed of the following (in $\mathrm{mM}$ ): $130 \mathrm{~K}$-gluconate, 4 $\mathrm{MgCl}_{2}, 1$ EGTA, 10 HEPES, $3 \mathrm{Na}_{2}$ ATP, 1 NaGTP, pH 7.2, 290 mOsm/ $\mathrm{kg}$. In the indicated experiments, the internal solution was supplemented with $0.25 \mathrm{~mm}$ purified clathrin and $0.2 \mathrm{~mm} 3 \mathrm{KDa}$-dextran conjugated to Texas Red (Life Technologies). External solution contained the following (in $\mathrm{mM}$ ): $130 \mathrm{NaCl}, 5 \mathrm{KCl}, 2 \mathrm{MgCl}_{2}, 10 \mathrm{HEPES}$ hemisodium salt, and 10 glucose, $\mathrm{pH}$ 7.4. The final $\mathrm{CaCl}_{2}$ concentration was always achieved by dilution from a $1 \mathrm{~m}$ stock solution and was $2 \mathrm{~mm}$ unless otherwise stated. All salts were from SigmaAldrich. Before the addition of glucose and $\mathrm{CaCl}_{2}$, the osmolality of the external solution was adjusted to $290 \mathrm{mOsm} / \mathrm{kg}$. All experiments were performed at room temperature $\left(23^{\circ} \mathrm{C}\right)$.

Recordings were made using an Axopatch-1D patch-clamp amplifier (Molecular Devices) under the control of a ITC-18 board (Instrutech) driven by WCP software (Dr. John Dempster, University of Strathclyde). Neurons were clamped at $-60 \mathrm{mV}$ and stimulated by a $1-2 \mathrm{~ms}$ depolar- ization step that drove membrane potential to $0 \mathrm{mV}$. The presence of functional autaptic synapses was identified by the generation of EPSCs, which were observed in $\sim 60 \%$ of the SCMs. Miniature EPSCs (mEPSCs) were either absent or occurred at a frequency $<1 \mathrm{~Hz}$. Further details of neurotransmission in SCMs have been described previously (PerezGonzalez et al., 2008; Albrecht et al., 2012).

To visualize how neuronal activity affected clathrin dynamics at synapses, SCMs expressing GFP-nLCa were imaged and simultaneously recorded. Coverslips were mounted on an RC-25 imaging chamber (Warner Instruments) and imaged using an inverted Olympus IX-71 microscope. An argon laser excited GFP-nLCa at $488 \mathrm{~nm}$ and fluorescence was acquired using q505LP dichroic and HQ535/50 m emission filters (Chroma). Images were collected through a LD Plan-Neofluar $40 \times, 0.6$ numerical aperture (NA) (Zeiss) or Plan Apo 60×, 1.45 NA (Olympus) objective and visualized on a EM-CCD C-9100 camera controlled by Hokawo software (Hamamatsu). Images from a $310 \times 310$ pixel region of interest (ROI) located on the dendritic tree were acquired at $3 \mathrm{~Hz}$. The somatic region was avoided because the high fluorescence levels precluded resolution of individual synapses. A transistor-transistor logic (TTL) pulse generated by WCP software controlled exposure time. Timing of laser light illumination was adjusted via a shutter (Uniblitz) to minimize photobleaching. In some experiments, synapses identified by staining and subsequent destaining with FM4-64 were recorded. In this particular condition, excitation was achieved via a 560/40× filter and fluorescence emission was collected with a 610LP filter (Chroma).

Analysis of electrophysiological and imaging data. Analysis was performed with custom-made macros written in Igor Pro software version 6.0 (Wavemetrics). Calculation of RRP size from $15 \mathrm{~Hz}$ trains was performed following the method described previously (Sakaba et al., 2002; Perez-Gonzalez et al., 2008). Briefly, current evoked by release of the RRP was obtained by the intercept on the ordinates axes of a linear fit to the steady-state phase of the cumulative plot of currents. Measurements of the RRP size were also done by local application of a hypertonic sucrose solution $(500 \mathrm{mOsm} / \mathrm{kg})$. This method measured the charge carried by EPSCs (in picocoulombs) and always provided larger estimates than the previous method. Therefore, the RRP size was defined by both approximations, allowing the establishment of low and high estimates (Albrecht et al., 2012).

Relative changes to basal GFP-nLCa fluorescence $\left(F_{\text {syn }} / F_{\text {syn_0 } 0}\right)$ were obtained for $1.3 \times 1.3 \mu \mathrm{m}$ ROIs centered on round, $\sim 1-1.5 \mu \mathrm{m}$ diameter structures where GFP-nLCa accumulated. Spots meeting this criterion were considered as synapses because immunostaining showed an almost complete colocalization of the construct with the vesicular proteins synaptophysin and VAMP-2. Typically, an imaging region contained two to seven putative synapses. This figure is in agreement with our electron micrographs of the dendritic tree of SCMs, where few consolidated synaptic contacts were obvious (data not shown, but see Furshpan et al., 1986). Puncta with an apparent diameter smaller than $1 \mu \mathrm{m}$ were not considered. Relative photobleaching $\left(F_{\text {dend }} / F_{\text {dend_0 } 0}\right)$ was estimated from dendritic regions without synapses. To report changes in synaptic fluorescence ( $\Delta F$ GFP-nLCa), we subtracted the average photobleaching of three different ROIs from relative changes in basal GFP-nLCa fluorescence as follows: $\Delta F=\left(F_{\text {syn }} / F_{\text {syn_0 } 0}\right)-\left(F_{\text {dend }} / F_{\text {dend_0 } 0}\right)$. Changes in fluorescence $(\Delta F)$ were only observed when stimulation evoked synaptic responses. In microcultures without functional autapses, $\Delta F=0$.

Correlative electrophysiology and optical or electron microscopy. At the end of a recording episode, neurons were micrographed, fixed in $4 \%$ paraformaldehyde, and stained with the appropriate primary antibodies. The following dilutions were used: CHC (X22, 1:2500; Thermo Scientific), synaptophysin (1:500; Synaptic Systems) and Bassoon (1:1000; Assay Designs). Overnight incubation with primary antibodies at $4^{\circ} \mathrm{C}$ was followed by staining with secondary antibodies labeled with Alexa Fluor 488, Alexa Fluor 555, or Alexa Fluor 647 (1:500; Invitrogen).

Immunostained cultures were viewed by wide-field microscopy in a Zeiss Axio examiner A1. Images were acquired with an Axiocam MRc5 using Axiovision software. Quantification of CHC levels in recorded microcultures was performed in ImageJ software by measuring the average fluorescence from the whole soma and nearby dendritic tree on an 
8-bit scale. To obtain relative $\mathrm{CHC}$ levels after knock down, acquisition settings were adjusted so that control somatodendritic regions provided fluorescence levels between 160 and 210 a.u. after subtracting background. No differences were found between estimates obtained by widefield and confocal microscopy.

The procedure used for correlative electrophysiology and electron microscopy experiments has been described previously (Albrecht et al., 2012). Briefly, neurons grown in thermanox coverslips (Nalgene) were micrographed and fixed in $2 \%$ glutaraldehyde. Microcultures were postfixed in $1 \%$ osmium tetroxide $/ 0.8 \%$ potassium ferricyanide, dehydrated, and embedded in EPON resin. Sections of recorded neurons were stained with uranyl acetate and lead citrate and viewed in a Jeol 1010 electron microscope.

Purification of clathrin from rat liver. CCVs were purified from rat liver following a method described previously (Campbell et al., 1984; Kaur et al., 2014). Briefly, three rat livers were washed in cold HKM buffer (25 mM HEPES, $\mathrm{pH}$ 7.4, $125 \mathrm{~mm}$ potassium acetate, $5 \mathrm{~mm}$ magnesium acetate, and $1 \mathrm{~mm}$ DTT), diced, made up to $40 \mathrm{ml}$ with HKM with protease mixture set III (catalog \#539134; Calbiochem), and homogenized in a dounce homogenizer. All steps were performed on ice or at $4^{\circ} \mathrm{C}$. The mixture was cleared by two centrifugation steps at $3800 \times g$. The supernatant was then ultracentrifuged at 45,000 rpm for $40 \mathrm{~min}$ in a $70 \mathrm{Ti}$ rotor $(149000 \times g)$. The resulting pellet was resuspended in $10 \mathrm{ml}$ of HKM, homogenized, and an equal volume of HKM containing Ficoll $(12.5 \%$ $\mathrm{w} / \mathrm{v})$ and sucrose $(12.5 \% \mathrm{w} / \mathrm{v})$ was added and mixed by inversion. The mixture was spun in a 70Ti rotor at 25,000 rpm for $20 \mathrm{~min}(46000 \times \mathrm{g})$. CCVs were in the supernatant, which was then diluted with 3 volumes of HKM and spun at 36,000 rpm for $60 \mathrm{~min}$ in a 70 Ti rotor $(96000 \times \mathrm{g})$. The pellet was resuspended in $15 \mathrm{ml}$ of HKM, homogenized, and left on ice for $20 \mathrm{~min}$. To pellet insoluble material, the suspension was spun at $17000 \times g$. Next, the supernatant was layered over a cushion of $8 \%(\mathrm{w} / \mathrm{v})$ sucrose/HKM (equal volume) and spun at 22,000 rpm $(\sim 80,000 \times g)$ in a swing-out rotor (SW41) for $2 \mathrm{~h}$. The pellet was resuspended in $0.5 \mathrm{ml}$ of uncoating buffer ( $1 \mathrm{M}$ Tris-HCl pH 7.4 $+1 \mathrm{~mm}$ DTT), rotated for $1 \mathrm{~h}$ at $4^{\circ} \mathrm{C}$, and vesicles pelleted out at $44,000 \mathrm{rpm}(96,000 \times \mathrm{g})$ in a Beckman MLA80 rotor for $25 \mathrm{~min}$. Samples were snap frozen in liquid nitrogen and stored at $-80^{\circ} \mathrm{C}$.

Statistical analysis. Data from averages were always expressed as mean \pm SEM. For statistical analysis, groups were compared using unpaired two-tailed Student's $t$ test. When comparing more than two groups, ANOVA analysis with Bonferroni's post hoc test was used. Significance level was set at $p<0.05$.

\section{Results \\ High-frequency stimulation reduces quantal amplitude and the size of the RRP in cholinergic SCMs}

For our experiments, we used SCMs (Fig. 1A) established from superior cervical ganglion neurons. This culture system allows cholinergic synapses to be studied in the absence of any other cell type and, because SCMs display a stereotyped neurotransmission (Perez-Gonzalez et al., 2008), short-term plasticity can be attributed exclusively to neuronal factors. We began by testing whether synaptic depression in SCMs was similar to other cholinergic synaptic systems. Application of a train of stimuli at $20 \mathrm{~Hz}$ frequency to a SCM evoked a decrease in quantal size that reverted in $\sim 15 \mathrm{~min}$ (Fig. 1B), comparable to results obtained at the neuromuscular junction (Naves and Van der Kloot, 2001). A maximal reduction of $25 \%$ in quantal amplitude was observed when $20 \mathrm{~Hz}$ stimulation periods lasted $2.5 \mathrm{~min}$. Quantal size was not affected by trains shorter than 1 min (Fig. 1C). Like quantal amplitude, the RRP was also affected after delivery of 3000 stimuli at $20 \mathrm{~Hz}$. The number of immediately available vesicles decayed by $\sim 30 \%$ and recovered within 15 min (Fig. $1 D$ ), again resembling previous findings in neuromuscular synapses (Wu and Betz, 1998). These results show that short-term depression mechanisms found at the neuromuscular junction are reproduced at
A

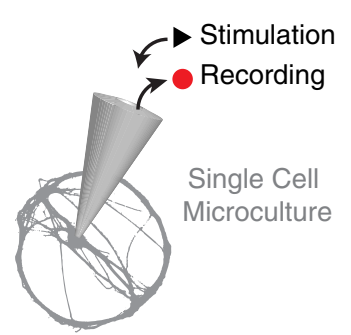

C

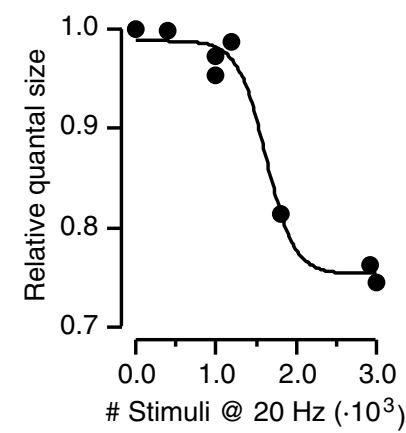

B
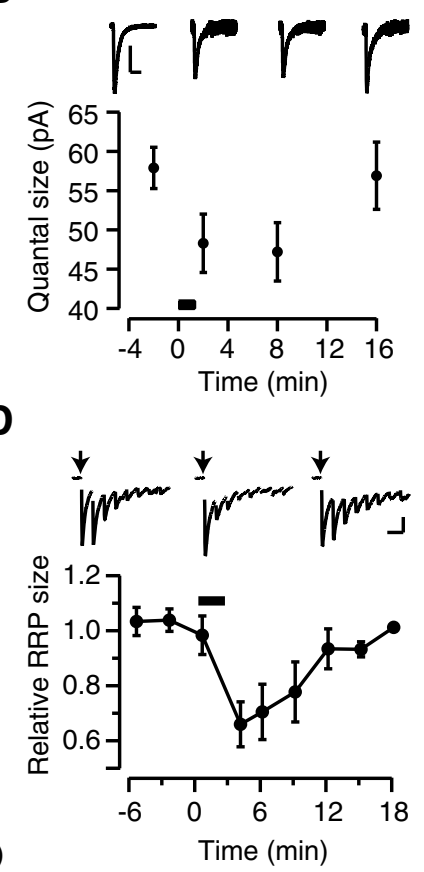

Figure 1. High-frequency stimulation reversibly reduces quantal size and the amount of available vesicles for release in cholinergic autaptic synapses. $A$, Schematic drawing showing the experimental setup used to record electrophysiological responses from SCMs. $\boldsymbol{B}, \mathrm{mEPSCs}$ were measured in an SCM before and after delivery of a train of 1600 stimuli applied at $20 \mathrm{~Hz}$. Recordings show average $\mathrm{mEPSC}$ before and 2,7, or $15 \mathrm{~min}$ after stimulation. Lower plot shows variations in quantal size relative to stimulus application (bar). Notice recovery of quantal size $15 \mathrm{~min}$ after the application of the train. Scale bars, 20 pA, 50 ms. C, Plot of relative changes in quantal size after $20 \mathrm{~Hz}$ application of the indicated number of stimuli. Each dot indicates a single neuron. Individual data were fitted by a sigmoid function, estimating a half reduction in quantal size at 1624 stimulations. $\boldsymbol{D}$, The RRP was reduced by 30\% after delivery of 3000 stimuli at $20 \mathrm{~Hz}$. Like quantal size, recovery occurred within $15 \mathrm{~min}$. The RRP was estimated from the cumulative analysis of synaptic responses obtained by applying 15 stimuli at $15 \mathrm{~Hz}$ (see Materials and Methods for details). Traces show EPSCs obtained before the application of a high-frequency train (left) and $3 \mathrm{~min}$ (middle) and $12 \mathrm{~min}$ (right) afterward. Each dot indicates mean \pm SEM $(n \geq 5)$. Scale bars, $1 \mathrm{nA}, 0.1 \mathrm{~s}$.

SCMs and validate this preparation for the investigation of cholinergic synapse plasticity.

\section{Clathrin moves out of the synapse upon stimulation}

Synaptic vesicle proteins move out of hippocampal presynaptic boutons upon high-frequency stimulation and are "followed" by clathrin presumably for extrasynaptic vesicle retrieval (Li et al., 2005; Fernández-Alfonso et al., 2006; Granseth et al., 2006). To determine whether clathrin also moves out of cholinergic synapses during high-frequency stimulation, SCMs were infected with lentiviruses to express GFP-nLCa, the neuronal isoform of clathrin light chain A tagged with enhanced GFP (Fig. 2A). The construct accumulated at presynaptic terminals, defined as $\sim 1$ to 1.5 - $\mu \mathrm{m}$-diameter structures that colocalized with the vesicular proteins synaptophysin and VAMP-2 (Fig. 2B). Simultaneous electrophysiology recording and fluorescence microscopy allowed us to assess the movement of clathrin from synapses after controlled stimulation. Upon application of a stimulus train, GFP-nLCa fluorescence decreased at presynaptic terminals and, at the same time, increased in perisynaptic regions (Fig. 2A). This transient change in GFP-nLCa fluorescence (Fig. 2C) likely reflects movement of the construct out of the synapse upon stimulation, as described previously for hippocampal boutons 


\section{A Experimental setup}
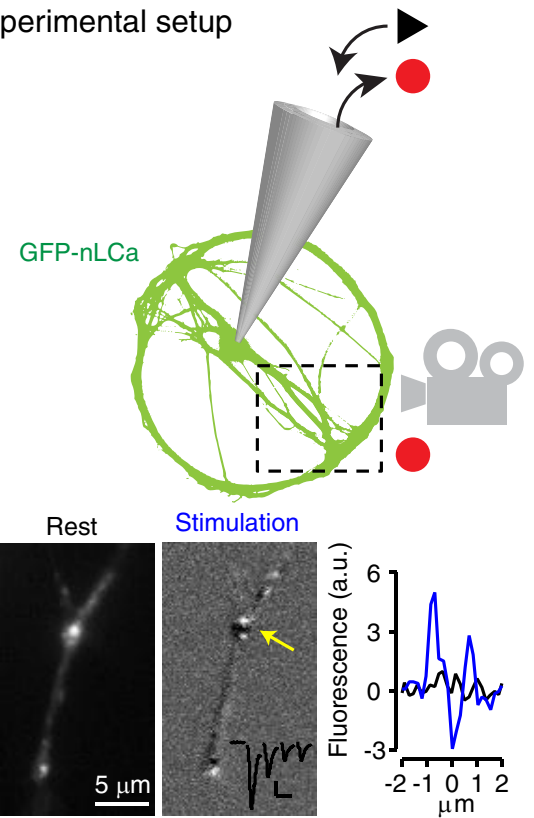

D
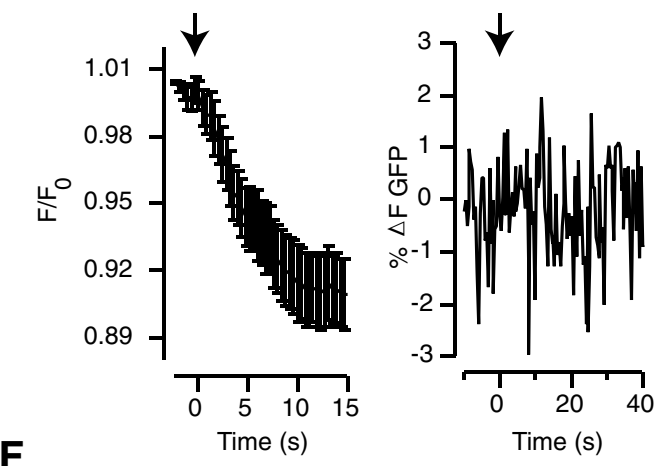

$\mathbf{F}$

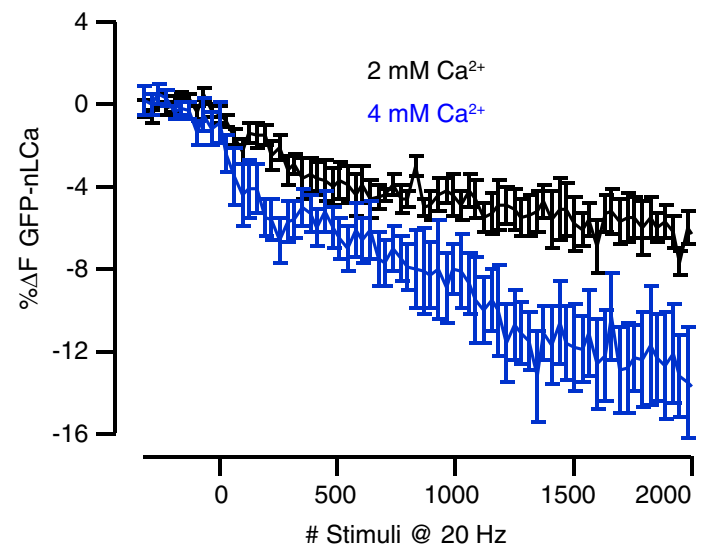

B
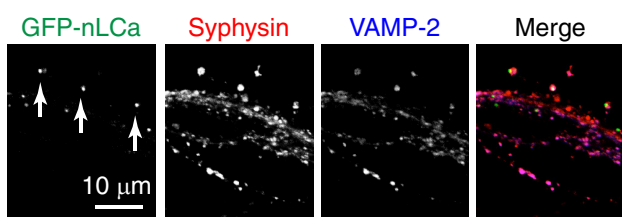

C

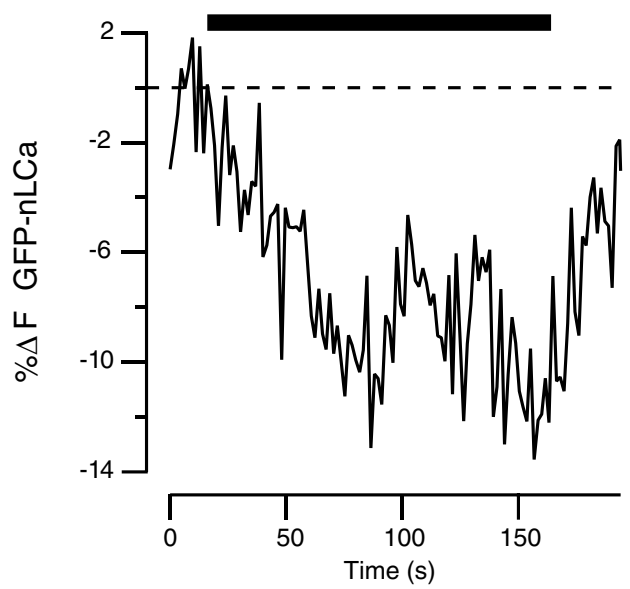

$\mathbf{E}$
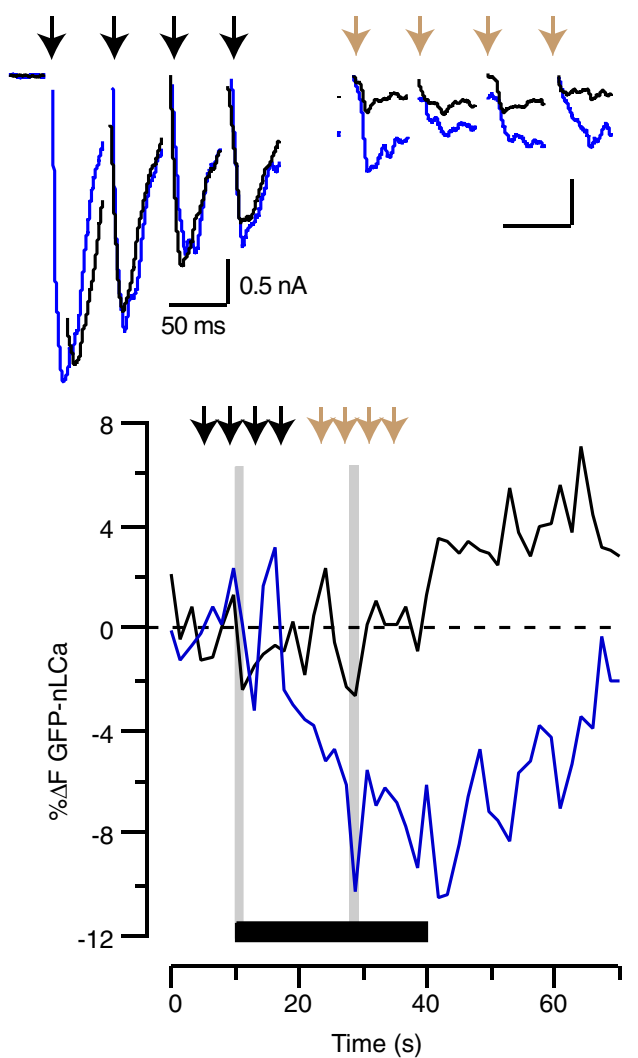

Figure 2. Clathrin moves out of synapses during high-frequency stimulation. $\boldsymbol{A}$, Schematic drawing of the methodology used. An example of GFP-nLCa mobility during sustained stimulation is shown at the bottom. Left, Image of two labeled puncta at rest. Middle, Changes in $n L C$ f fluorescence are shown by subtracting an average baseline image. Notice fluorescence moved (white) away from putative synapses (black). Right, Plot profile of changes in fluorescence at the arrowed bouton before (black) and during (blue trace) stimulation. Scale bars, $0.5 \mathrm{nA}, 50 \mathrm{~ms}$. B, GFP-nLCa accumulates in synapses. Dendrites of a SCM where GFP-nLCa is expressed in discrete puncta (arrows). Labeled structures colocalize with the presynaptic markers synaptophysin and VAMP-2. C, Monitoring changes in GFP-nLCa fluorescence of putative synapses during high-frequency stimulation. Fluorescence intensity decreased during stimulus application and reverted immediately afterwards. Bar shows delivery of 3000 stimuli at $20 \mathrm{~Hz}$. D, Left, Example of a neuron in which synapses were first identified by sequential staining and destaining of FM4 - 64 (arrow indicates application of a train of stimuli). A mask of the ROIs defining the position of synapses was placed over the image of cytoplasmic GFP expression. Right, GFP fluorescence did not change during the application of atrain of stimuli (arrow shows onset). $\boldsymbol{E}$, The decrease in GFP-nLCa fluorescence was dependent on release probability, evidenced by changing $\left[\mathrm{Ca}^{2+}\right]_{\text {ext }}$ from $2 \mathrm{~mm}$ (black) to $4 \mathrm{~mm}$ (blue). The change in $\left[\mathrm{Ca}^{2+}\right]_{\text {ext }}$ had little effect on the initial EPSCs of the stimulus train (black arrows), but consistently evoked larger synaptic responses as depression developed (brown arrows). The change in release probability affected differentially the time course of relative changes in GFP-nLCa fluorescence in a synapse of the recorded neuron. Although no changes were obvious during application of 600 stimuli at 20 $\mathrm{Hz}$ (bar) in $2 \mathrm{~mm}\left[\mathrm{Ca}^{2+}\right]_{\text {ext }}$ (black), a reversible decrease in fluorescence occurred in $4 \mathrm{~mm}\left[\mathrm{Ca}^{2+}\right]_{\text {ext }}$. F, Average change in GFP-nLCa fluorescence relative to the number of stimuli delivered at $20 \mathrm{~Hz}$ as a function of $\left[\mathrm{Ca}^{2+}\right]_{\text {ext }}$. Differences between $2 \mathrm{~mm}\left[\mathrm{Ca}^{2+}\right]_{\text {ext }}(n=14$ cells $)$ and $4 \mathrm{~mm}\left[\mathrm{Ca}^{2+}\right]_{\text {ext }}(n=13$ cells $)$ appeared as stimulation proceeded. Traces indicate mean \pm SEM. 
(Granseth et al., 2006). However, it is possible that GFP fluorescence is quenched due to a decrease in synaptic $\mathrm{pH}$, because it has been shown that synaptic terminals acidify during highfrequency stimulation (Rossano et al., 2013). To test this possibility, we measured changes in cytoplasmic GFP in presynaptic terminals, identified by staining with FM4-64 and subsequent stimulus-dependent destaining (Fig. 2D). These experiments showed that the fluorescence of GFP located at synaptic boutons was not quenched during high-frequency stimulation and suggest that the decrease in GFP-nLCa fluorescence is due solely to movement out of the synapse.

If a decrease in GFP-nLCa fluorescence were associated with synaptic vesicle retrieval, it should depend on the extent of exocytosis. To evaluate this possibility, we next investigated the change in synaptic GFP-nLCa fluorescence as a function of release probability, shown by increasing $\left[\mathrm{Ca}^{2+}\right]_{\text {ext }}$ from 2 to $4 \mathrm{~mm}$. In the example shown in Figure $2 E$, there were no obvious changes in GFP-nLCa fluorescence during application of 600 stimuli at $20 \mathrm{~Hz}$ in $2 \mathrm{~mm}\left[\mathrm{Ca}^{2+}\right]_{\mathrm{ext}}$. However, a significant decrease occurred when $\left[\mathrm{Ca}^{2+}\right]_{\text {ext }}$ was changed to $4 \mathrm{~mm}$. The enhancement of release probability had little effect at the beginning of the train, but had a significant contribution later (see recordings in Fig. 2E, top) as more stimuli were delivered. On average, long-lasting, high-frequency stimulation of synaptic terminals operating at a low release probability evoked a decrease in presynaptic clathrin levels of $\sim 5 \%$ after 2000 stimuli delivered at 20 $\mathrm{Hz}$ (Fig. $2 \mathrm{~F}$ ). In contrast, application of the same stimulation paradigm to synapses operating at a high release probability decreased presynaptic clathrin by $\sim 15 \%$. Based on these results, we wondered whether the transient decrease in clathrin availability contributes to synaptic depression and, if so, how?

\section{Lowering clathrin levels decreases synaptic currents}

To investigate whether clathrin availability constrains neurotransmission, we needed to establish a method to manipulate clathrin levels experimentally to mimic the synaptic clathrin loss observed during stimulation. To do this, we reduced the levels of CHC in SCMs using lentiviral infection that coupled shRNA production with expression of GFP. Control experiments in which neurons expressed GFP alone or GFP + GL2 shRNA showed normal clathrin levels (Fig. 3A). Putative synapses, as shown by synaptophysin staining, were positively labeled for $\mathrm{CHC}$. Therefore, cytoplasmic overexpression of GFP did not interfere with clathrin localization to synapses (Fig. $3 B$ ). Delivery of $\mathrm{CHC}$ shRNA homogeneously reduced clathrin levels through the cytoplasm and a comparable knock down was found at soma and synapses (Fig. 3C). Due to the major role of clathrin in synaptic vesicle recycling, a possible effect of $\mathrm{CHC}$ knock down on synaptic vesicle proteins was evaluated. In control infections, VAMP-2 and synaptophysin appeared at a density of $0.09 \pm 0.02(n=6)$ and $0.05 \pm 0.02(n=5)$ puncta $\cdot \mu \mathrm{m}^{-2}$, respectively. Similar densities of $0.07 \pm 0.02(n=9)$ VAMP-2 and $0.04 \pm 0.02(n=$ 10) synaptophysin puncta $\cdot \mu \mathrm{m}^{-2}$ were observed after reduction of clathrin levels. Therefore, it is unlikely that the expression of CHC RNAi affects the levels of synaptic-vesicle-associated proteins. However, there were large individual variations in clathrin depletion by RNAi. Some neurons showed normal CHC levels, whereas others had almost no label. We therefore investigated each neuron individually by correlating two methods: electrophysiology and immunofluorescence microscopy (Fig. 4A). Once synaptic responses were measured in infected neurons, SCMs were micrographed, immunostained for $\mathrm{CHC}$, and subsequently identified.
A

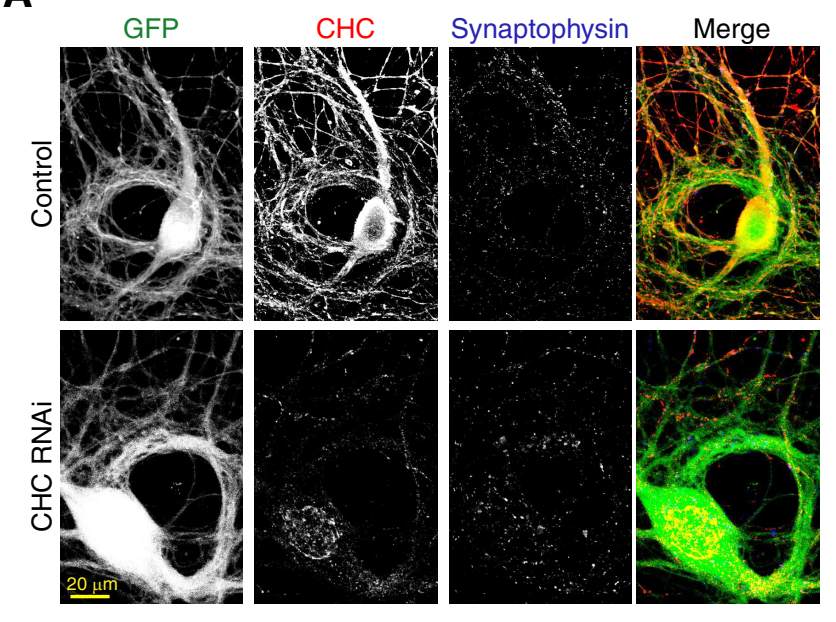

B
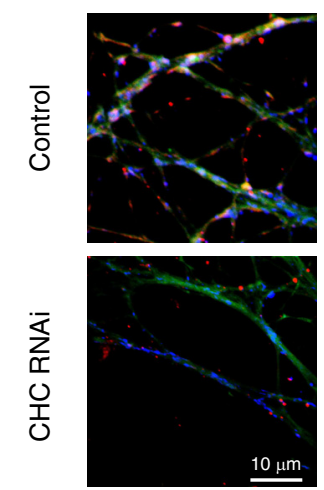

C

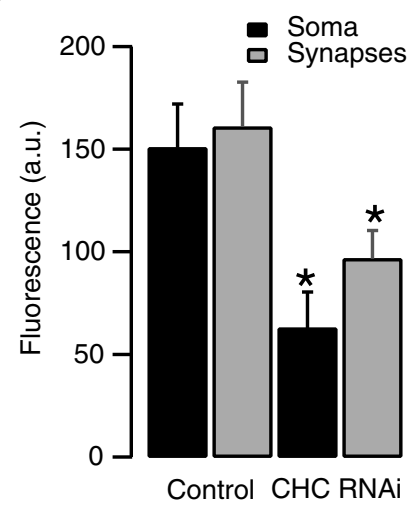

Figure 3. Depletion of $\mathrm{CHC}$ by RNAi in SCMs. A, Representative fluorescence micrographs showing single GFP-expressing SCMs costained for $\mathrm{CHC}$ (red) and synaptophysin (blue). SCMs were infected with lentiviruses to express GFP alone (control) or GFP and shRNA to deplete CHC (CHC RNAi). $\boldsymbol{B}$, Expanded view of micrographs in $\boldsymbol{A}$ to show that clathrin was decreased at synapses after RNAi. C, Bar chart showing quantification of $\mathrm{CHC}$ levels at the cell soma or synapses (identified by synaptophysin staining). Bars indicate mean $\pm \operatorname{SEM}(n \geq 5)$. Asterisks denote significant differences $(p<0.05)$.

The first observation was an apparent reduction in EPSCs as the clathrin concentration decreased (Fig. 4B). To investigate how synaptic responses were affected by clathrin levels, binned data from all recorded cells $(n=134)$ were well described by a sigmoidal function relating EPSC amplitude and CHC levels (Fig. $4 C)$. On average, synaptic responses transitioned from $\sim 0.6 \mathrm{nA}$ in cells with low clathrin levels to $\sim 2 \mathrm{nA}$ in cells with normal clathrin levels, displaying a [clathrin $]_{\text {half }}$ value of 0.78 . Therefore, an $\sim 20 \%$ decrease in clathrin levels reduced the amplitude of the average EPSC by 35\%. No synaptic responses were found when clathrin levels were reduced by $>80 \%$, likely reflecting the impossibility of establishing or maintaining functional neurotransmission in such conditions.

Decreased synaptic currents in cells with lowered clathrin levels are due to a presynaptic effect

The depressed synaptic currents observed after clathrin depletion could be due to a presynaptic or postsynaptic mechanism. To distinguish these possibilities, we first evaluated presynaptic plasticity by paired-pulse stimulation. Control neurons showed a characteristic paired-pulse ratio of $\sim 1$ for a $1 \mathrm{~s}$ interval, as described previously (Perez-Gonzalez et al., 2008; Albrecht et al., 2012). As expected for a primarily presynaptic mechanism, knock 
A Experimental setup

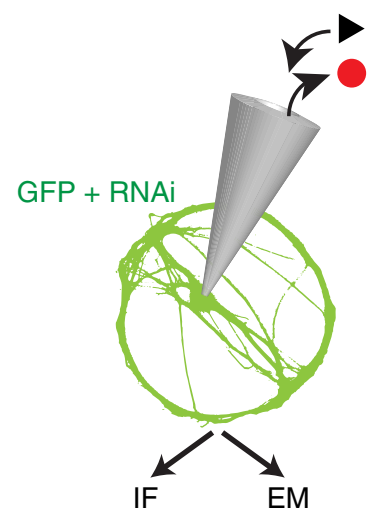

C

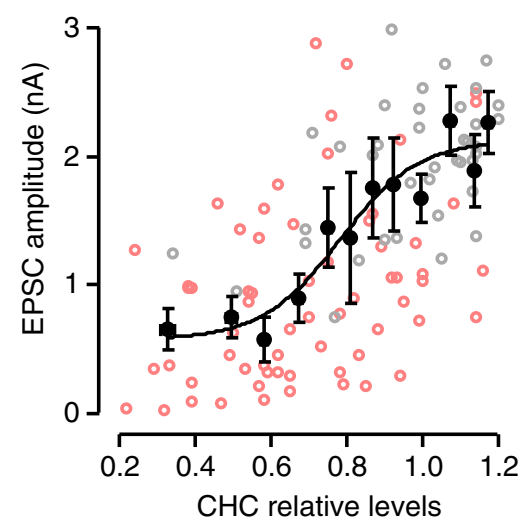

E

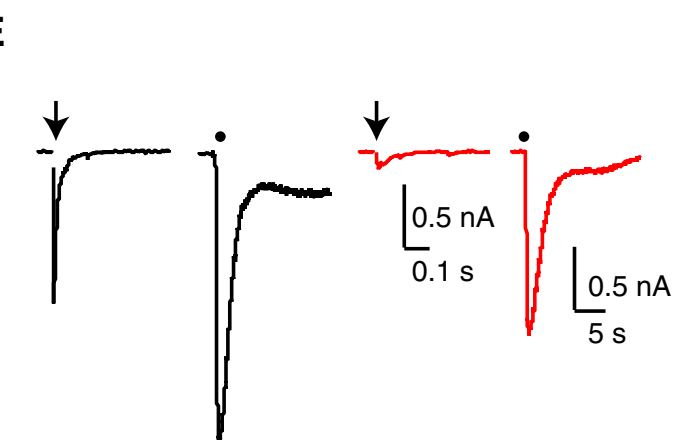

B

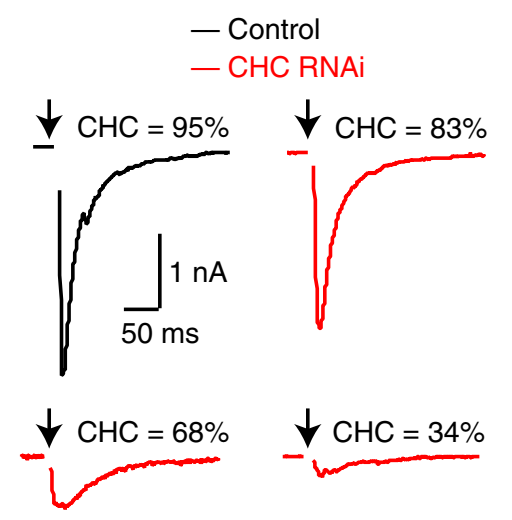

D

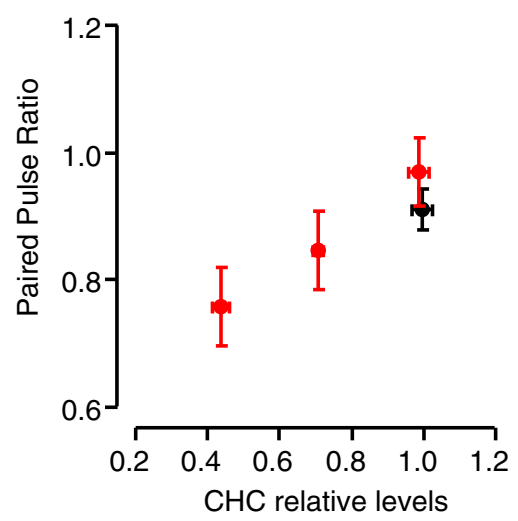

$\mathbf{F}$

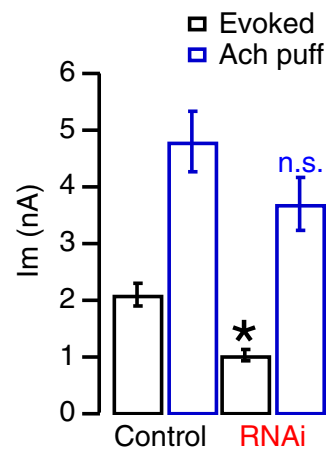

Figure 4. Synaptic transmission depends on clathrin levels. A, Summary of the methodology applied for this figure and subsequent work. Correlative experiments relating electrophysiological recordings and immunofluorescence (IF) or electron microscopy (EM) were performed for SCMs. B, The amplitude of EPSCs was correlated to $\mathrm{CHC}$ levels measured by IF. Example recordings from four different neurons with different clathrin levels; single sample events are shown. $C$, A sigmoid function described the relationship between the amplitude of evoked synaptic currents and $\mathrm{CHC}$ levels. Data were grouped in bins (dots, $n=10$ ). Each open circle represents mean EPSC amplitude of a single neuron. Notice the large variation from neurons in which $\mathrm{CHC}$ was depleted. Error bars indicate SEM. D, Presynaptic plasticity, evaluated as paired-pulse ratio obtained for a $1 \mathrm{~s}$ time interval, is related to $\mathrm{CHC}$ levels. $\boldsymbol{E}$, Presynaptic terminals are more sensitive than postsynaptic terminals to $\mathrm{CHC}$ knock down. Sample recordings from a control and a knock-down neuron to compare the sensitivity of presynaptic and postsynaptic terminals with a decrease in clathrin levels. First, a postsynaptic response was evoked by a $1 \mathrm{~ms}$ depolarization of the presynaptic terminal (arrow). Next, a $50 \mathrm{~ms}$ puff of $1 \mathrm{~mm}$ acetylcholine was applied on top of the SCM (dot) to evoke a postsynaptic response, with no requirement of the presynaptic component. $\boldsymbol{F}$, Knock down of $\mathrm{CHC}$ significantly decreased $(p<0.05)$ the amplitude of EPSCs evoked by electrical stimulation, but did not affect responses generated by local application of acetylcholine. Control neurons, $n=7$; CHC-depleted neurons, $n=11$.

down of clathrin was associated with the paired-pulse ratio: those neurons displaying the lowest clathrin levels also showed the strongest depression, but in neurons in which clathrin depletion failed, plasticity was normal (Fig. 4D). A second line of evidence was obtained by comparing, in the same neuron, synaptic responses evoked by single depolarization or by an acetylcholine puff. Postsynaptic responses triggered by an electrical stimulus involve the release of acetylcholine from presynaptic terminals, whereas the response to local application of neurotransmitter only requires the postsynaptic element. EPSCs generated chemically were larger than those evoked electrically (Fig. 4E), suggesting that postsynaptic receptors are not saturated by neurotransmitter release from a single $\mathrm{AP}$ and that local application of $1 \mathrm{~mm}$ acetylcholine provides a readout of the functional pool of postsynaptic nicotinic receptors present in a SCM. On average, knock down of $\mathrm{CHC}$ significantly reduced the amplitude of evoked EPSCs, but did not have an effect on responses generated by local delivery of acetylcholine (Fig. 4F). Together, these experiments show that the function of presynaptic terminals was particularly sensitive to $\mathrm{CHC}$ depletion. In contrast, postsynaptic terminal function remained largely unaffected. These results indicate that the abundance of clathrin constrains neurotransmission via a presynaptic action.

\section{Lowering clathrin levels reduces the RRP of vesicles}

To gain an understanding of the effects of low clathrin levels on presynaptic terminal function, we next investigated the functional organization of synaptic vesicles. It is considered that vesicles are distributed in two large pools: the RRP, which is responsible for fast vesicle release, and the cytoplasmic pool, which acts as a reservoir and is organized in several subpools (Denker and Rizzoli, 2010). Because the RRP critically determines synaptic responses and short-term plasticity, we applied two complementary methodologies to investigate the size of this vesicle pool: a $15 \mathrm{~Hz}$ train of stimuli and the local application of hypertonic solution to obtain low and high estimates, respectively (Albrecht et al., 2012). The first method, performing cumulative analysis of synaptic responses during sustained stimulation (Fig. $5 A$ ), showed that the release of the whole RRP in a control neuron would, on average, evoke a postsynaptic response of $4.3 \mathrm{nA}$. Considering that exocytosis of a single vesicle triggers a postsynaptic current of $\sim 60 \mathrm{pA}$, a lower limit for the RRP from all autapses of an average SCM would be $\sim 70$ vesicles. The second method (Fig. $5 B$ ) relied on the analysis of the postsynaptic response evoked by a local puff of a hypertonic solution. Because the charge carried by evoked EPSCs was $447 \mathrm{pC}$, and taking into 
A
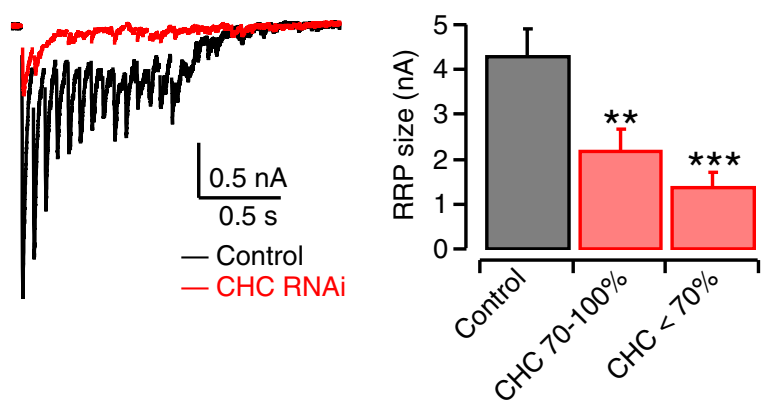

B
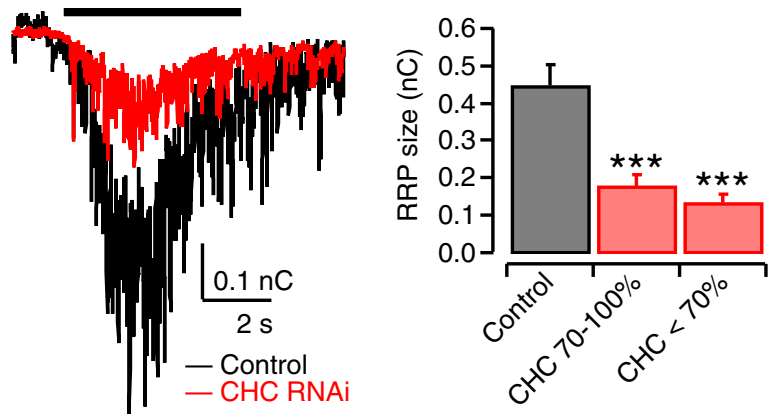

C

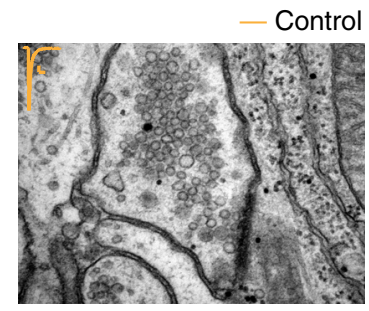

D

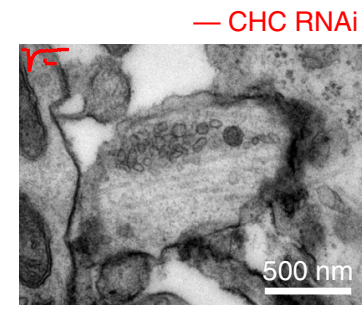

Figure 5. The size of the RRP of vesicles is highly sensitive to a decrease in clathrin levels. $\boldsymbol{A}$, Representative traces estimating the size of the RRP based on the application of a train of 15 stimuli delivered at $15 \mathrm{~Hz}$. Note that depression of synaptic responses was enhanced when $\mathrm{CHC}$ was depleted (red). Bar chart summarizing the mean \pm SEM size of the RRP. ${ }^{* *} p<0.01$; ${ }^{* * *} p<0.001$. $\boldsymbol{B}$, A second method to estimate the size of the RRP was based on a 5 s application of a hyperosmotic shock. Example traces and summary bar chart are shown as in $A$. C, D, Electron micrographs illustrating the appearance of the morphological RRP. Control synapses typically showed several vesicles docked at active zones (C). In contrast, CHC RNAi terminals displayed less vesicles attached to their active zones and some appeared completely devoid of vesicles (D). Corresponding synaptic responses for this neuron are shown on the top left corner. Scale bars, $0.2 \mathrm{nA}, 100 \mathrm{~ms}$.

account that an average mEPSC carried $\sim 2 \mathrm{pC}$, the RRP could thus be as high as $\sim 220$ vesicles. Assuming that axosomatic autapses are the main contributors to synaptic responses due to dendritic filtering properties (Bekkers and Stevens, 1996), and considering that an average SCM would contain $\sim 25$ axosomatic contacts (Albrecht et al., 2012), the RRP of a prototypical autapse would be defined by three to nine synaptic vesicles. This figure is comparable to other small conventional synapses, such as hippocampal boutons (Schikorski and Stevens, 1997).

The RRP size was very sensitive to small decreases in clathrin levels. Due to the large variability in $\mathrm{CHC}$ knock down, clathrin levels were classified in two groups: moderate knock down (70$100 \%$ relative to control CHC levels) and intense knock down $(<70 \%)$. Under moderate CHC knock down, the RRP was already reduced to approximately one-half, ranging from 41 to 90 vesicles ( $p<0.01$ and $p<0.001$, respectively), and the RRP decreased to approximately one-third under intense $\mathrm{CHC}$ knock

down, within a range of 22 to 67 vesicles $(p<0.001)$. Because an apparently small reduction of presynaptic clathrin levels decreased RRP size by $50 \%$, we deemed that such an effect should be obvious by morphological analysis. Assuming that the functional RRP is tightly related to the number of docked vesicles at active zones, we correlated synaptic responses to ultrastructural analysis. Three control SCMs were investigated, which had an average EPSC amplitude of $1.6 \mathrm{nA}$. For comparison, five SCMs in which clathrin levels were reduced by CHC RNAi were also studied. Because growth on Thermanox coverslips precluded doing immunofluorescence on neurons selected for electron microscopy, $\mathrm{CHC}$ knock down was estimated from EPSC amplitudes. Selected neurons had synaptic potencies ranging from 0.1 to $0.8 \mathrm{nA}$, so their clathrin levels were likely within the range of intense knock down (i.e., $<70 \%$ of control levels; Fig. $4 C$ ).

Active zones found in random sections of control neurons showed a characteristic appearance (Fig. 5C). Typically, two to six synaptic vesicles were clustered in presynaptic densities indicating sites of preferential release. Under CHC knock down, presynaptic terminals consistently showed fewer docked vesicles (Fig. $5 D$ ) and some densities appeared to be devoid of synaptic vesicles. Although it would be necessary to reconstruct the entire active zone to certify a complete absence of vesicles, this observation fits to functional estimates of RRP size assuming that the number of synaptic contacts was not affected by $\mathrm{CHC}$ knock down. Again, considering an average of $\sim 25$ axosomatic synapses on a prototypical SCM, an intense decrease of clathrin levels would reduce the RRP from zero to three vesicles in a single autapse. This is consistent with the ultrastructural observations shown here.

\section{Lowering clathrin levels reduces the density and size of synaptic vesicles}

We next evaluated synaptic vesicle populations morphologically in neurons from which we had previously recorded synaptic responses. Synaptic terminals (Fig. 6A) contained numerous cholinergic, clear vesicles that coexisted with a small proportion of large dense-core vesicles (LDCVs). Each type of vesicle has a different biogenesis: clear vesicles essentially bud from endosomes and LDCVs are sorted in the trans-Golgi network and transported to synaptic terminals (Bonanomi et al., 2006; Zhang et al., 2010). We found that each type of vesicle had a different sensitivity to manipulation of clathrin levels. There was an obvious reduction in the density of clear vesicles $(p<0.05)$ in all $\mathrm{CHC}$ RNAi synapses studied, as shown in the examples of axosomatic and axodendritic contacts (Fig. 6B). Two factors contributed to this decrease (Fig. 6C). First, there was a reduction from $60 \pm 9$ vesicles in control autapses $(n=27)$ to $25 \pm 4$ vesicles $(n=21)$ in knock-down boutons. Second, there was an increase in terminal area after CHC RNAi from $0.68 \pm 9$ to $0.90 \pm 0.13 \mu \mathrm{m}^{2}$. However, the increase in presynaptic surface did not affect the density of LDCVs, which remained unaltered after CHC depletion (Fig. 6D).

Further analysis addressed the overall distribution of synaptic vesicles throughout the cytoplasm. Measurement of distance to the nearest portion of plasma membrane provided a readout of vesicle position relative to putative release sites. Distance of vesicles to the plasma membrane was similar in control and low clathrin levels (Fig. 6E). For example, the proportion of vesicles within $50 \mathrm{~nm}$ from the presynaptic membrane, which were considered docked, was $5.6 \%(n=1366)$ and $7 \%(n=728)$ in control and CHC RNAi conditions, respectively. No differences were observed in the distance of LDCVs to the plasma membrane. 


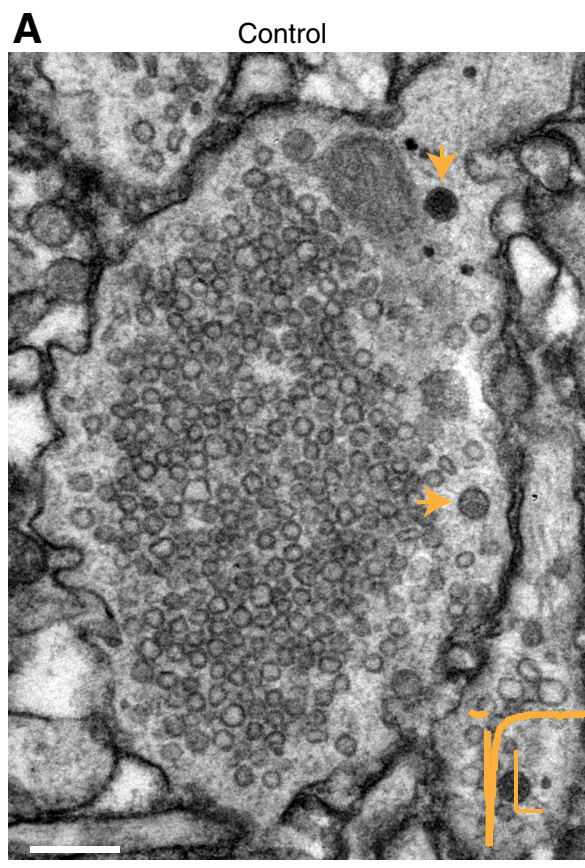

B
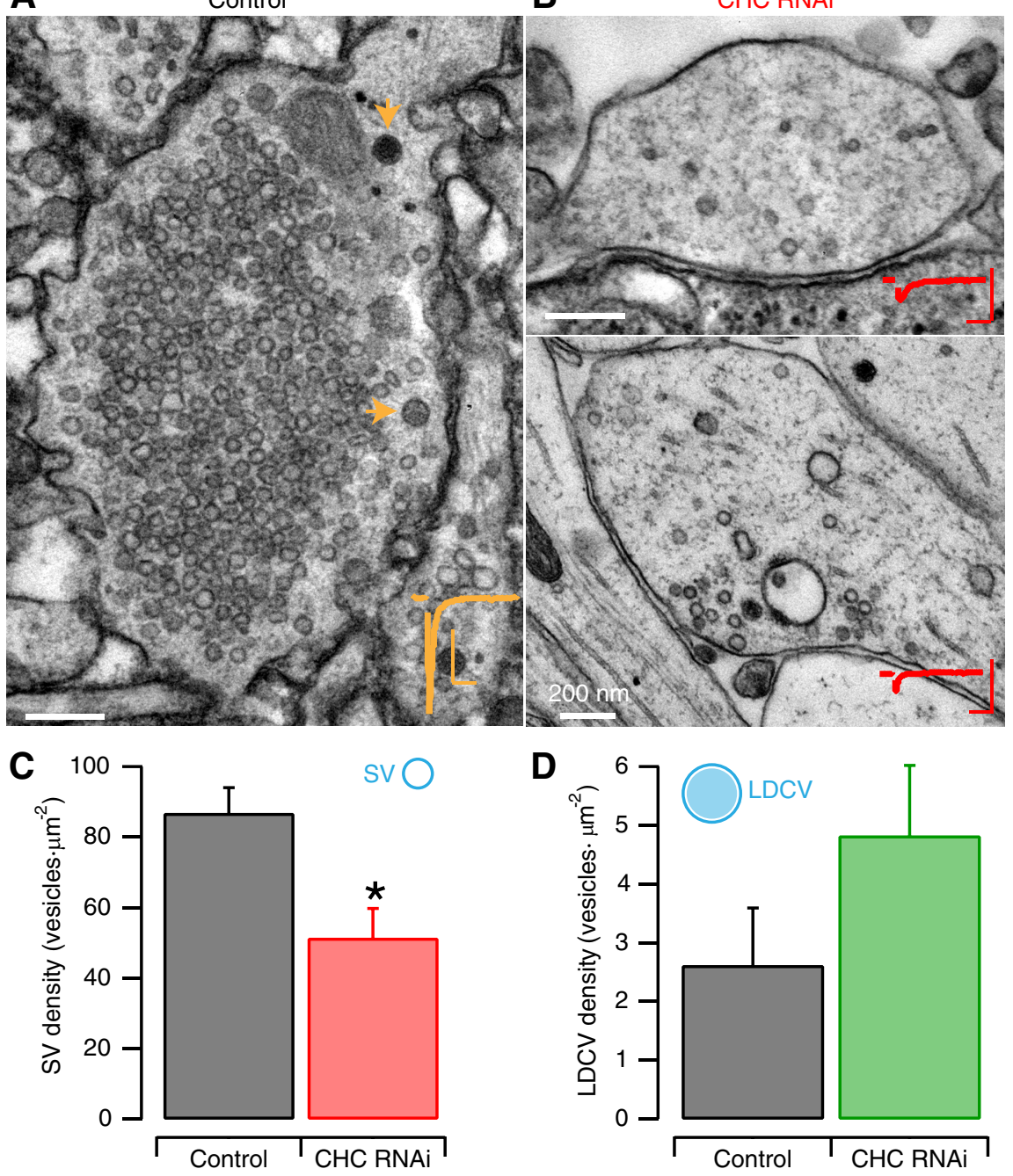

E

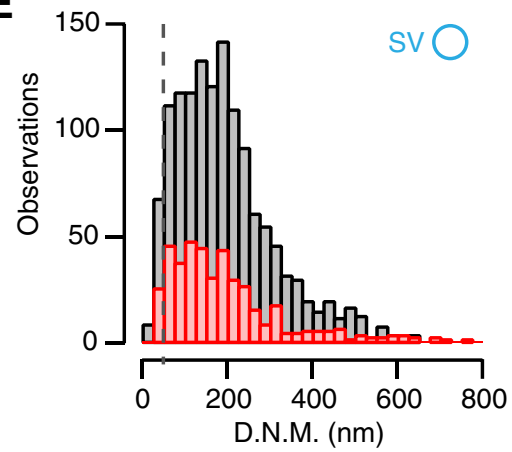

D

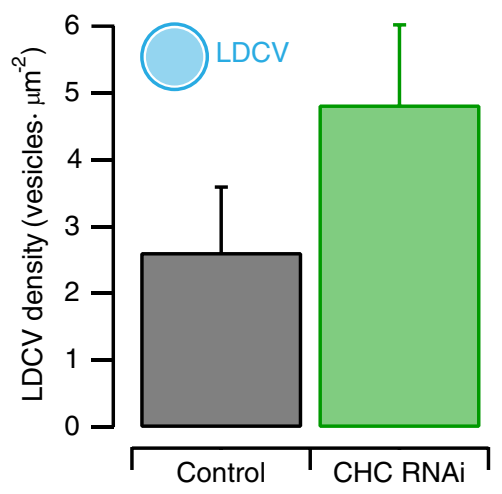

F

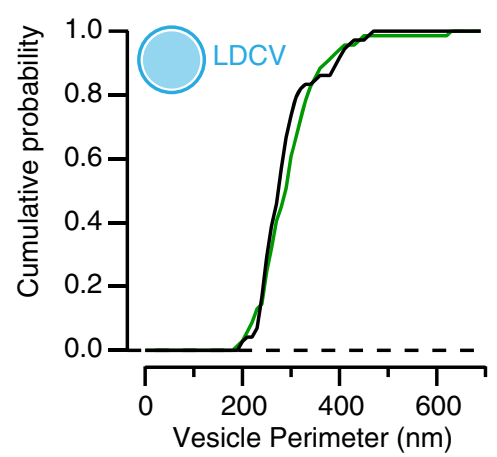

Figure 6. Effect of CHC knock down on clear and LDCVs. A, Electron micrograph of an axosomatic presynaptic terminal from a control neuron. Corresponding EPSC from this neuron is shown in orange. The cytoplasm is filled with clear synaptic vesicles, and a few LDCVs are observed (arrows). Scale bars, $1 \mathrm{nA}, 100 \mathrm{~ms}$. B,. Micrographs of axosomatic (top) and axodendritic (bottom) synapses from two different neurons in which $\mathrm{CHC}$ was depleted. The associated synaptic responses are shown in red. Scale bars, $1 \mathrm{nA}, 100 \mathrm{~ms}$. C, D, Bar chart showing the average density of clear synaptic vesicles $(\boldsymbol{C})$ and LDCVs $(\boldsymbol{D})$ in control and CHC RNAi presynaptic terminals. Mean \pm SEM is shown. ${ }^{*} p<0.05$. $\boldsymbol{E}$, The cytoplasmic distribution of clear synaptic vesicles was not affected by $\mathrm{CHC}$ knock down. Dotted line indicates distance to nearest membrane (D.N.M.) of $50 \mathrm{~nm}$ or docked vesicles. $\boldsymbol{F}$, Cumulative distribution of LDCV size showing that a decrease in clathrin levels did not change the perimeter of this type of vesicle.

Therefore, there was a proportional reduction of synaptic vesicles without apparently affecting their distribution in low clathrin levels. This finding suggests equilibrium between cytoplasmic pools and the RRP (see Discusion).
A striking finding was that clear synaptic vesicles were smaller when clathrin levels were reduced. This was in contrast to LDCVs, the size of which was not affected (Fig. 6F). The distribution of vesicle sizes under $\mathrm{CHC}$ knock down was left shifted with respect to controls (Fig. 7A). A potential explanation for this observation was that, after CHC knock down, CME was not operative and vesicles were being reformed by alternative mechanisms. This possibility was minimized, because clathrin-coated structures forming at the plasma membrane were observed both in normal and low clathrin levels (Fig. $7 B$ ), suggesting that, under conditions of reduced clathrin, the synaptic vesicles that reform are abnormal.

On average, the diameter of clear synaptic vesicles was reduced by $\sim 5-8 \mathrm{~nm}$ in low clathrin levels, similarly to a previous finding in $C$. elegans with a temperaturesensitive mutant of CHC (Sato et al., 2009). Such a decrease could potentially affect neurotransmission, because changing the mean vesicle diameter from 53 to 48 $\mathrm{nm}$ would reduce the potential volume to store neurotransmitter by $\sim 25 \%$. In summary, the overall reduction of synaptic vesicle pools (Figs. 5, 6) and vesicle size (Fig. $7 A$ ) observed by CHC RNAi demonstrates a high sensitivity of clear vesicles to changes in clathrin levels.

\section{Knock down of $\mathrm{CHC}$ reduces quantal size}

The possible functional implications of a reduction in synaptic vesicle density and size after CHC knock down were further investigated by analyzing spontaneous synaptic activity. On average, $0.44 \pm 0.1$ mEPSCs $s^{-1}$ were observed in control SCMs (Fig. 7C). Knock down of CHC reduced the frequency of spontaneous events, albeit with large variability among control cells that precluded observing significant differences. When clathrin levels were moderately reduced, between $70 \%$ and $100 \%$ of wild-type levels, mEPSCs were observed at a frequency of $0.28 \pm 0.1$ $\mathrm{s}^{-1}$ (Fig. $7 D$ ). For clathrin levels $<70 \%$ of control conditions, mEPSCs appeared at $0.18 \pm 0.1 \mathrm{~s}^{-1}$. Such a reduction could be attributed to the overall decrease in synaptic vesicles, which was observed by electron microscopy (Fig. 6A-C). However, mEPSC amplitude was also affected by CHC knock down (Fig. 7E). Quantal size of control mEPSCs was well described by a single Gaussian fit with a mean value of $72 \mathrm{pA}$. An intense reduction of clathrin levels $<70 \%$ of wild-type values generated a new population of events that were, again, well distributed by a single Gaussian fit to a mean amplitude of $14 \mathrm{pA}$. Both populations were 
A

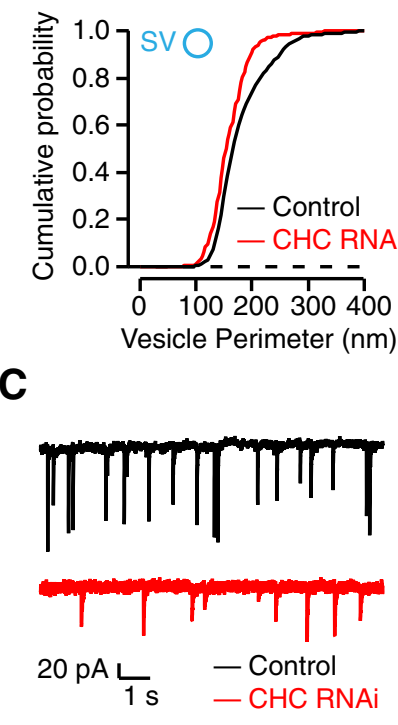

B

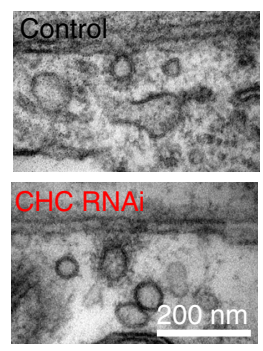

D

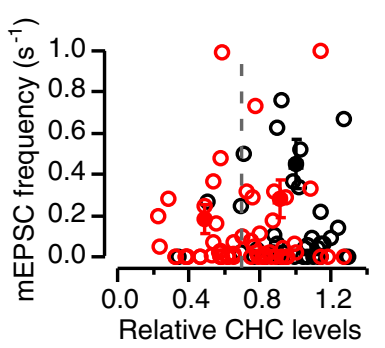

E
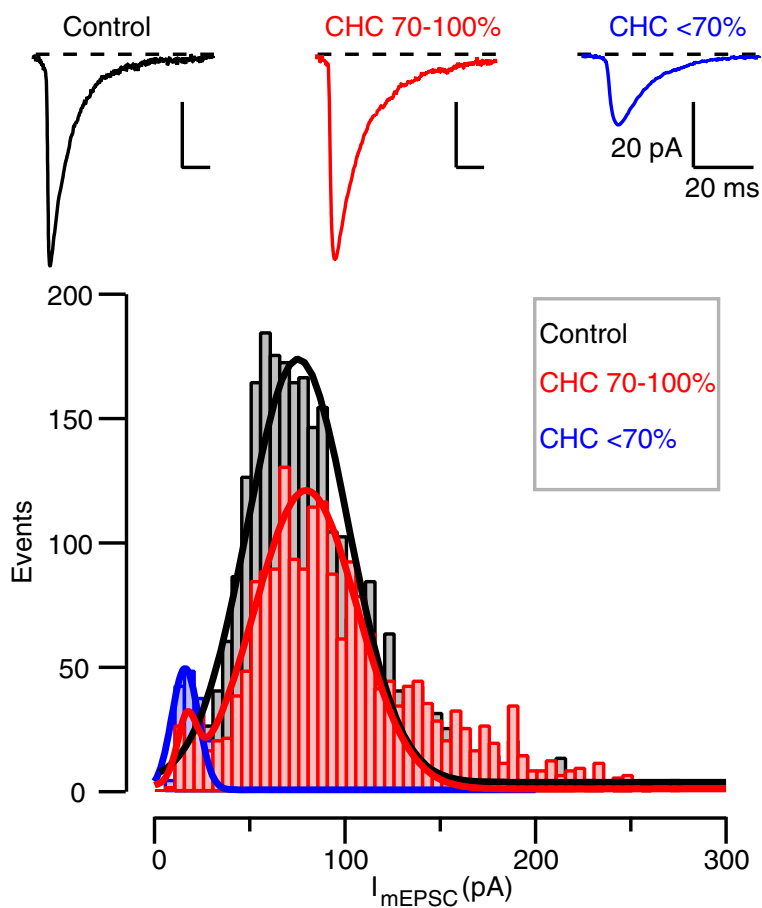

Figure 7. Knock down of $\mathrm{CHC}$ reduces the size of clear synaptic vesicles and decreases the amplitude of miniature spontaneous events. $\boldsymbol{A}$, Cumulative distribution of the perimeter of clear synaptic vesicles. Knock down of $\mathrm{CHC}$ left shifted the size of this vesicle type. $0 \mathrm{n}$ average, the diameter of clear vesicles was $5-8 \mathrm{~nm}$ smaller when clathrin levels were reduced. $\boldsymbol{B}$, Electron micrographs showing CCVs attached to the plasma membrane occurred at synapses in cells with control or low clathrin levels. C, Sample recordings of spontaneous activity in control (black) and CHC knock-down conditions. $\boldsymbol{D}$, The frequency of mEPSCs was reduced when clathrin levels were decreased. Open circles indicate the average mEPSC frequency for individual control (black) or knock-down (red) neurons. Black dot shows mean \pm SEM of all control experiments. Dashed line indicates the criterion for grouping knock-down neurons in two sets (red dots, mean \pm SEM) as a function of relative $\mathrm{CHC}$ content: cells with 70-100\% control CHC levels (moderate knock down) and cells $<70 \%$ (intense knock down). $\boldsymbol{E}$, Effect of $\mathrm{CHC}$ depletion on quantal size. Top examples indicate the average mEPSC for three different neurons in control conditions (black) and after moderate (red) or intense (blue) CHC knock down. The amplitude distribution of mEPSCs obtained in control neurons and after intense CHC knock down was well fitted by a single Gaussian with mean values of 72 and $14 \mathrm{pA}$, respectively. In contrast, the distribution of $\mathrm{mEPSC}$ amplitudes for a moderate CHC knock down was fitted by two Gaussians, with similar mean amplitudes to control and intense knock-down populations. obvious when clathrin levels were moderately decreased. Under this condition, most mEPSCs fell within the control population, but $\sim 8 \%$ of the events were found within the group of low quantal size. An intense reduction in clathrin levels was thus required to consistently modify quantal size. This observation contrasts with the high sensitivity of the RRP to moderate changes in clathrin levels, as described previously (Fig. 5).

A consistent shrinkage of $5-8 \mathrm{~nm}$ in vesicle diameter would decrease the available vesicle volume to store acetylcholine between $\sim 25 \%$ and $\sim 30 \%$. However, analysis of mEPSCs indicates that, in cells with low clathrin levels, the quantal size was actually reduced by $80 \%$. It is thus likely that, in addition to the reduction in vesicle volume, lowering $\mathrm{CHC}$ levels alters the composition of synaptic vesicles. Inadequate retrieval of specific cargo such as vesicular ACh transporters or proton pumps that may lead to impaired loading of neurotransmitter could explain the additional reduction in quantal size beyond that predicted by morphological data.

\section{Reversibility of clathrin depletion effects by dialysis of exogenous clathrin}

Our experimental approach, using RNAi to mimic the physiological reduction in clathrin availability, has the drawback that the effects seen may be due solely to lowering clathrin levels and may be due in part to an off-target effect of RNAi. We set out to "rescue" the RNAi phenotype by dialyzing purified clathrin into the cell (Fig. 8A). This allowed us to test the reversibility of the described presynaptic changes after $\mathrm{CHC}$ knock down in the same neuron by recording after breaking in whole-cell configuration and again after 15-20 min of dialysis with exogenous clathrin. Two specific effects of $\mathrm{CHC}$ depletion reverted after dialysis. First, the amplitude of EPSCs increased (cf. Figs. $8 B, 4 C)$. In $63 \%$ of the neurons studied $(n=11)$, the size of EPSCs was at least doubled and, on average, synaptic responses increased by a factor of 2.6. We confirmed that this change in EPSC amplitude was due to a presynaptic increase in clathrin availability rather than a postsynaptic effect because responses to local puffs of $1 \mathrm{~mm}$ Ach did not change before and after clathrin dialysis $(n=4)$. Second, the frequency of spontaneous events increased from $0.22 \pm 0.1$ to $1.54 \pm 0.7(n=5$; cf. Figs. $8 C, 7 D)$. Infusion of exogenous clathrin did not induce the appearance of mEPSCs in neurons without spontaneous activity. Contrary to mEPSC frequency, quantal size was, on average, unaltered. The amplitude of mEPSCs did not change, being $42 \pm 1 \mathrm{pA}(n=427)$ before and $41 \pm 1 \mathrm{pA}(n=919)$ after $20 \mathrm{~min}$ of dialysis with exogenous clathrin.

Together, our results suggest that presynaptic clathrin levels are a limiting factor for presynaptic function. The ability to dialyze clathrin allowed us to test this idea directly. We repeated the experimental paradigm in Figure $1 D$ supplementing control cells with exogenous clathrin to test whether physiological clathrin depletion was participating in the depression of synaptic responses after high-frequency stimulation. RRP size was no longer affected by the application of 3000 stimuli delivered at $20 \mathrm{~Hz}$ (Fig. $8 D$ ). An overall enhancement of neurotransmitter release probability was obvious as dialysis time increased, such that there was an almost twofold increase in the RRP size 20 min after breaking into whole-cell configuration. In contrast, changes in quantal size after high-frequency stimulation (Fig. $1 B, C$ ) were difficult to interpret. Clathrin dialysis gradually widened the distribution of mEPSC amplitudes; however, the low spontaneous activity of SCMs precluded a detailed population analysis. Our results suggest that continuous supplementation of presynaptic 

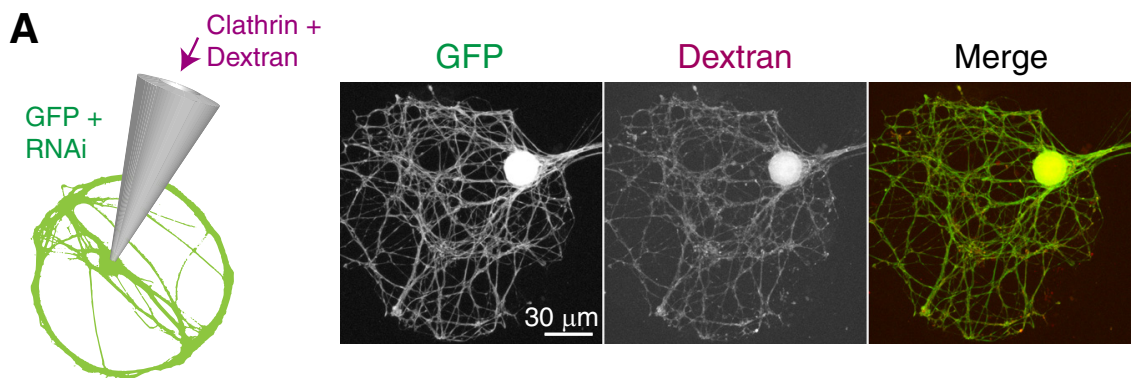

B

- CHC RNAi

- Control

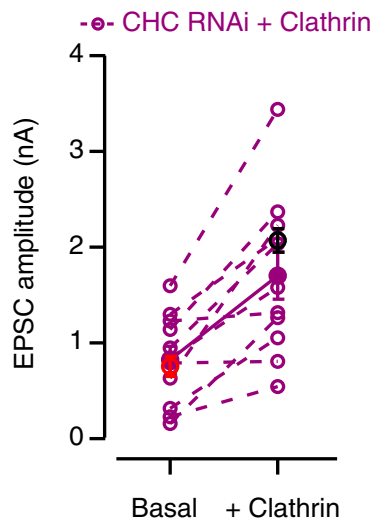

C

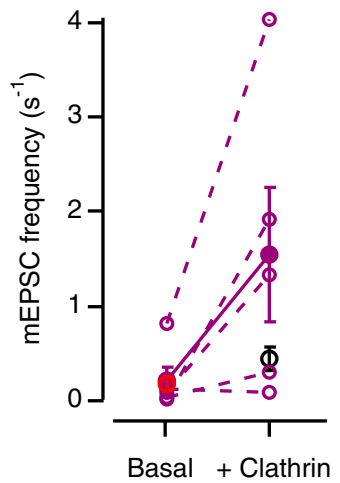

D
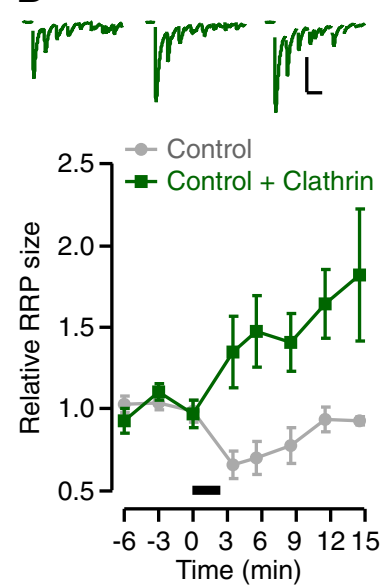

Figure 8. Reversibility of clathrin depletion phenotype by dialysis of exogenous clathrin. $\boldsymbol{A}$, Summary of the methodology applied. Internal solution was supplemented with $0.25 \mathrm{~mm}$ purified rat clathrin and $0.2 \mathrm{~mm}$ Texas red/3 kDa dextran. Successful dialysis was verified by visualizing the neuritic tree of GFP-expressing neurons (representative micrographs are shown). $\boldsymbol{B}$, The decrease in EPSC amplitude caused by RNAi-mediated clathrin depletion reverted after 15-20 min dialysis of exogenous clathrin. Broken lines show the change in EPSC amplitude for individual neurons. Notice that, on average, the infusion of exogenous clathrin allowed neurons to recover control synaptic potency (compare dots with red and black circles, all indicating mean \pm SEM). $\boldsymbol{C}$, Same as $\boldsymbol{B}$ but showing the effect of clathrin dialysis on the frequency of spontaneous events. $\boldsymbol{D}$, Infusion of exogenous clathrin in control cells (green) protected against the decrease in the RRP induced by sustained high-frequency stimulation. Control data (gray) are from Figure 1D. Bar indicates application of 3000 stimuli at $20 \mathrm{~Hz}$. Scale bars, $1 \mathrm{nA}, 100 \mathrm{~ms}$.

clathrin levels increases variability in the composition and/or size of synaptic vesicles reformed by endocytosis.

\section{Discussion}

The present study tested whether clathrin levels are a limiting factor for synaptic function. Our results showed that synaptic clathrin availability is not constant and decreases during periods of neuronal activity as a function of stimulus strength and presynaptic release probability. Synaptic responses are related to clathrin levels by a sigmoid function, such that clathrin decreases of $>20 \%$ significantly reduce synaptic strength. Beyond this threshold, terminals become particularly sensitive and the organization of synaptic vesicle pools, vesicle size, and quantal content are all decreased. These findings indicate that presynaptic clathrin levels are a dynamic property that contributes to plasticity when synapses are subjected to sustained physiological stimulation (Fig. 9).

Lateral movement of synaptic proteins after exocytosis is restricted to the periactive zone, where clathrin coat formation initiates (Heuser and Reese, 1973; Shupliakov et al., 1997). As CME proceeds with a time constant of $\sim 15$ s (Wu et al., 2007), exocytosis and endocytosis are balanced at low-frequency stimulations. However, during sustained stimulation, the exocytic load overcomes endocytic capacity. This results in an excess of synaptic vesicle proteins incorporated to the plasma membrane that

move far from active zones (Saheki and De Camilli, 2012). In hippocampal boutons, synaptic cargo laterally diffusing in the plasma membrane is tracked by clathrin (Granseth et al., 2006). Similarly, our results in cholinergic autapses suggest that clathrin moves out of synapses upon stimulation. Movement is reversible and dependent on release probability, so the amount of clathrin available at synapses is not fixed and transiently decreases upon stimulation. We estimate that delivery of 2000 stimuli at $20 \mathrm{~Hz}$ causes between $\sim 5 \%$ and $\sim 15 \%$ of total clathrin to move out of the terminal depending on whether synapses operate at normal or high release probability. However, movement of clathrin out of synapses would only define a lower limit to the actual decrease of clathrin levels. The formation of clathrin coats themselves, or the capacity of synaptic vesicles to act as a clathrin buffer, further decreases the ACP (Denker et al., 2011). Altogether, the combination of our results and previous work, suggest that synapses- especially those operating at a high release probability-are likely to undergo a transient clathrin "knock down" when subjected to intense stimulation. Two pieces of experimental evidence reinforce this possibility. First, the addition of exogenous clathrin prevents depletion of the RRP of vesicles after high-frequency stimulation (Fig. 8D). Second, transient decreases in clathrin levels obtained by CHC knock down mirror synaptic depression features observed after sustained stimulation (Figs. 4, 5, 7). So the question is, to what extent does clathrin availability influence presynaptic function? In absolute terms, a $20 \%$ decrease in clathrin levels is expected to be on the order of $10-100$ $\mu \mathrm{M}$. This estimate can be obtained from recovery experiments. Considering axonal tortuosity, the high diffusion coefficient of clathrin (Nandi and Wahl, 1988), and the fact that half of endogenous clathrin remained after depletion, presynaptic [clathrin] is unlikely to be $>0.1-0.2 \mathrm{~mm}$. In any case, such a moderate decrease in clathrin levels would be the threshold value for presynaptic terminals to acquire a depressed phenotype. However, two considerations should be taken into account: (1) our results based on RNAi take place in the timescale of days, and (2) all presynaptic changes are only occurring together when clathrin levels are intensely reduced. By just crossing the $20 \%$ threshold, only synaptic strength and RRP size are affected.

The first consideration can be addressed by comparison to previous works, such as those making a genetic acute effect on endocytosis using shibire flies. When these flies are driven to nonpermissive temperature, synaptic transmission is immediately impaired, caused by the arrest of clathrin-coated pits at the plasma membrane after block of dynamin-mediated endocytosis (Koenig and Ikeda, 1989). Interestingly, continuous stimulation of neuromuscular junctions in these mutant flies at nonpermissive temperature generates a similar phenotype to the described CHC knock-down effects: synaptic strength and RRP size gradu- 


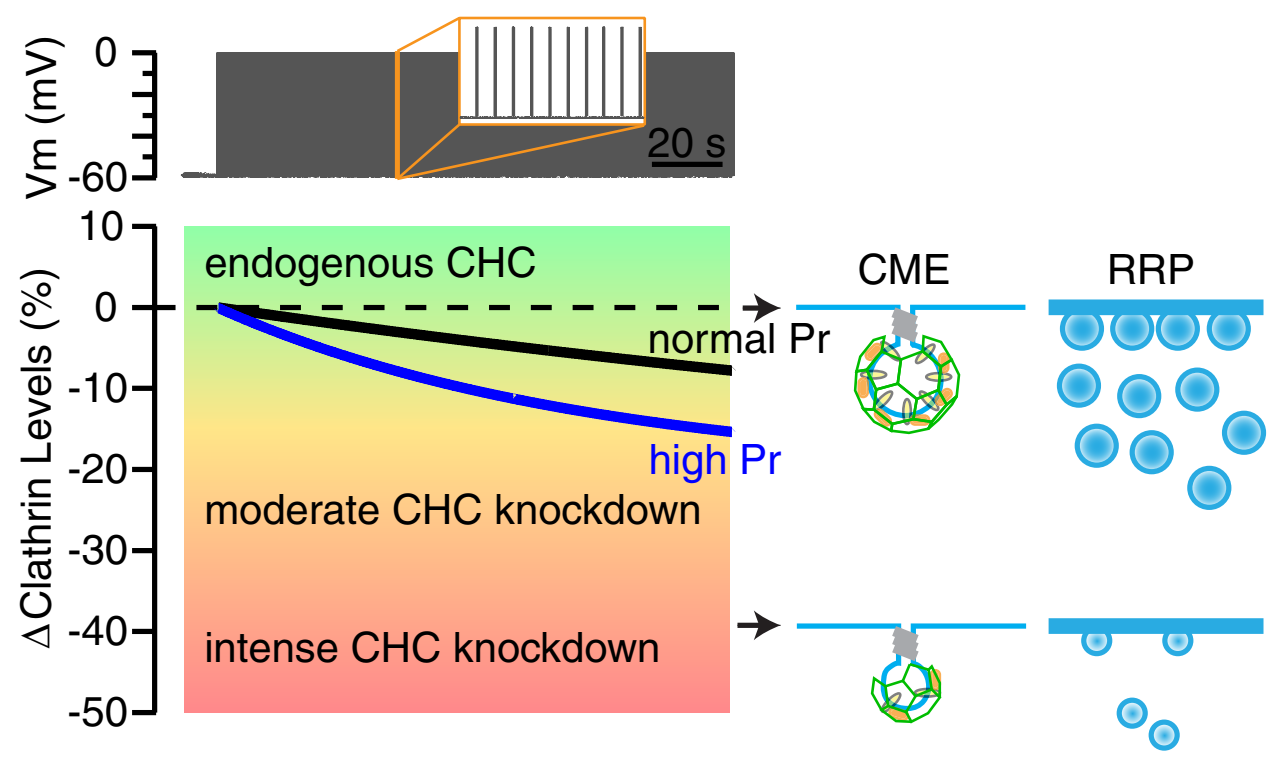

Figure 9. Summary model of the contribution of presynaptic levels to synaptic depression. In synapses with low levels of clathrin, the quality of vesicles that are retrieved is inferior compared with vesicles retrieved in conditions of normal clathrin abundance. The vesicles are smaller and carry less neurotransmitter and the pools of vesicles are also reduced. We found that application of a sustained, high-frequency stimulation triggers the movement of clathrin out of presynaptic terminals, reducing presynaptic clathrin levels. Examples shown are average traces from normal $\left(2 \mathrm{~mm}\left[\mathrm{Ca}^{2+}\right]_{\text {ext }}\right.$, black) or high $\left(4 \mathrm{~mm}\left[\mathrm{Ca}^{2+}\right]_{\text {ext }}\right.$, blue) neurotransmitter release probability conditions. This implies that the fidelity of vesicle reformation decreases during stimulation such that the final few vesicles to be retrieved convey less information than those retrieved at the beginning of activity. This compromise in synaptic transmission contributes to synaptic depression.

ally decrease (Li and Schwarz, 1999). Similar observations are obtained after FlAsH-FALI-mediated protein photoinactivation of clathrin light chain (Heerssen et al., 2008). These acute effects are best explained if the RRP and cytoplasmic pool of vesicles are in equilibrium, suggesting endocytosis as a direct determinant of synaptic transmission by maintaining the size of synaptic vesicle pools. This view is also supported by synaptophysin-pHluorin imaging of hippocampal boutons, which shows that CME is key to synaptic strength (Granseth and Lagnado, 2008). However, an alternative role for clathrin can be proposed from the acute action of two endocytosis inhibitors on synaptic release of hippocampal boutons (Hua et al., 2013). These experiments reveal that CME is involved in the clearance of vesicular debris at the active zone during high-frequency stimulation, which appears to be an important determinant of short-term depression. Whether CME is required to maintain synaptic vesicle pools or is necessary for a correct function of active zones during sustained stimulation, both mechanisms support a pivotal role of clathrin. In this scenario, even an apparently minor decrease in presynaptic clathrin levels would lead to a reduction in synaptic strength and could be potentially implicated in the generation of shortterm depression.

The second consideration can be addressed when stimulation conditions proceed to a more marked decrease in presynaptic clathrin levels (i.e., <30\%). In this situation, a reduction in quantal size would also contribute to short-term depression. The effect is specific for clear synaptic vesicles, showing that clathrin is essentially dispensable for LDCV biogenesis. This finding is not surprising because LDCVs are sorted in the trans-Golgi network and transported to axon terminals, whereas clear SVs recycle via presynaptic endosomes and contain proteins with numerous clathrin motifs (Edwards, 2007; Jung and Haucke, 2007). A possible cause of the decrease in quantal size is defective CME, as suggested by the presence of smaller vesicles in low clathrin levels. Clathrin-coated intermediates were obvious in knock- down conditions, suggesting reduced clathrin availability as a putative cause affecting the fidelity of CME. Lower levels of available clathrin triskelia would have an effect on all reactions involving CME (Rizzoli, 2014), probably favoring the smallest cage geometries. As a result, smaller vesicles would be formed (Cheng et al., 2007), likely with an altered composition. For example, the expected copies of vesicle-associated proteins (Takamori et al., 2006) would be reduced, which could contribute to a decrease in quantal size. These phenomena will affect vesicles being retrieved at the plasma membrane, but also those vesicles formed from bulk endocytosis, which invaginates large regions of plasma membrane, creating bulk endosomes from which synaptic vesicles can bud (Cousin, 2009).

This view should, however, be reconciled with results obtained after acute application of clathrin, which rescued evoked neurotransmission and increased mEPSC frequency but did not lead to an increase of quantal size. This raises the possibility that clathrin required a longer time period to recover this particular aspect of the depleted phenotype or that the effect on quantal size was due to an off-target effect of RNAi. Overall, our experiments dialyzing clathrin into SCMs to cause the rescue of clathrin depletion or enhanced function in control conditions suggest that clathrin levels define a limit for presynaptic function. This also implies that other endocytic proteins, e.g. stonin 2 (Willox and Royle, 2012) are amply abundant. The stoichiometry of clathrin to adaptors in a CCV is $\sim 3: 1$ (Borner et al., 2012), so adaptor supply would be unlikely to become an issue even under intense activity.

In conclusion, our results establish clathrin levels as a dynamic property of presynaptic terminals, meaning that the availability of clathrin for vesicle retrieval is key to high-fidelity neurotransmission and is implicated directly in short-term synaptic depression. 


\section{References}

Albrecht D, López-Murcia FJ, Pérez-González AP, Lichtner G, Solsona C, Llobet A (2012) SPARC prevents maturation of cholinergic presynaptic terminals. Mol Cell Neurosci 49:364-374. CrossRef Medline

Balaji J, Ryan TA (2007) Single-vesicle imaging reveals that synaptic vesicle exocytosis and endocytosis are coupled by a single stochastic mode. Proc Natl Acad Sci U S A 104:20576-20581. CrossRef Medline

Bekkers JM, Stevens CF (1996) Cable properties of cultured hippocampal neurons determined from sucrose-evoked miniature EPSCs. J Neurophysiol 75:1250-1255. Medline

Bonanomi D, Benfenati F, Valtorta F (2006) Protein sorting in the synaptic vesicle life cycle. Prog Neurobiol 80:177-217. CrossRef Medline

Borner GH, Antrobus R, Hirst J, Bhumbra GS, Kozik P, Jackson LP, Sahlender DA, Robinson MS (2012) Multivariate proteomic profiling identifies novel accessory proteins of coated vesicles. J Cell Biol 197:141-160. CrossRef Medline

Campbell C, Squicciarini J, Shia M, Pilch PF, Fine RE (1984) Identification of a protein kinase as an intrinsic component of rat liver coated vesicles. Biochemistry 23:4420-4426. CrossRef Medline

Cheng Y, Boll W, Kirchhausen T, Harrison SC, Walz T (2007) Cryoelectron tomography of clathrin-coated vesicles: structural implications for coat assembly. J Mol Biol 365:892-899. CrossRef Medline

Cousin MA (2009) Activity-dependent bulk synaptic vesicle endocytosis-a fast, high capacity membrane retrieval mechanism. Mol Neurobiol 39: 185-189. CrossRef Medline

Denker A, Rizzoli SO (2010) Synaptic vesicle pools: an update. Front Synaptic Neurosci 2:135. CrossRef Medline

Denker A, Kröhnert K, Bückers J, Neher E, Rizzoli SO (2011) The reserve pool of synaptic vesicles acts as a buffer for proteins involved in synaptic vesicle recycling. Proc Natl Acad Sci U S A 108:17183-17188. CrossRef Medline

Doxsey SJ, Brodsky FM, Blank GS, Helenius A (1987) Inhibition of endocytosis by anti-clathrin antibodies. Cell 50:453-463. CrossRef Medline

Edwards RH (2007) The neurotransmitter cycle and quantal size. Neuron 55:835-858. CrossRef Medline

Fernández-Alfonso T, Kwan R, Ryan TA (2006) Synaptic vesicles interchange their membrane proteins with a large surface reservoir during recycling. Neuron 51:179-186. CrossRef Medline

Furshpan EJ, Landis SC, Matsumoto SG, Potter DD (1986) Synaptic functions in rat sympathetic neurons in microcultures. I. Secretion of norepinephrine and acetylcholine. J Neurosci 6:1061-1079. Medline

Goud B, Huet C, Louvard D (1985) Assembled and unassembled pools of clathrin: a quantitative study using an enzyme immunoassay. J Cell Biol 100:521-527. CrossRef Medline

Granseth B, Lagnado L (2008) The role of endocytosis in regulating the strength of hippocampal synapses. J Physiol 586:5969-5982. CrossRef Medline

Granseth B, Odermatt B, Royle SJ, Lagnado L (2006) Clathrin-mediated endocytosis is the dominant mechanism of vesicle retrieval at hippocampal synapses. Neuron 51:773-786. CrossRef Medline

Heerssen H, Fetter RD, Davis GW (2008) Clathrin dependence of synapticvesicle formation at the Drosophila neuromuscular junction. Curr Biol 18:401-409. CrossRef Medline

Heuser JE, Reese TS (1973) Evidence for recycling of synaptic vesicle membrane during transmitter release at the frog neuromuscular junction. J Cell Biol 57:315-344. CrossRef Medline

Hua Y, Woehler A, Kahms M, Haucke V, Neher E, Klingauf J (2013) Blocking endocytosis enhances short-term synaptic depression under conditions of normal availability of vesicles. Neuron 80:343-349. CrossRef Medline

Jung N, Haucke V (2007) Clathrin-mediated endocytosis at synapses. Traffic 8:1129-1136. CrossRef Medline

Kaur S, Fielding AB, Gassner G, Carter NJ, Royle SJ (2014) An unmet actin requirement explains the mitotic inhibition of clathrin-mediated endocytosis. Elife 3:e00829. CrossRef Medline

Koenig JH, Ikeda K (1989) Disappearance and reformation of synaptic ves- icle membrane upon transmitter release observed under reversible blockage of membrane retrieval. J Neurosci 9:3844-3860. Medline

Li J, Schwarz TL (1999) Genetic evidence for an equilibrium between docked and undocked vesicles. Philos Trans R Soc Lond B Biol Sci 354: 299-306. CrossRef Medline

Li Z, Burrone J, Tyler WJ, Hartman KN, Albeanu DF, Murthy VN (2005) Synaptic vesicle recycling studied in transgenic mice expressing synaptopHluorin. Proc Natl Acad Sci U S A 102:6131-6136. CrossRef Medline

Miller TM, Heuser JE (1984) Endocytosis of synaptic vesicle membrane at the frog neuromuscular junction. J Cell Biol 98:685-698. CrossRef Medline

Moskowitz HS, Yokoyama CT, Ryan TA (2005) Highly cooperative control of endocytosis by clathrin. Mol Biol Cell 16:1769-1776. CrossRef Medline

Nandi P, Wahl P (1988) Diffusion properties of clathrin on the surface of isolated mouse liver nuclei by the fluorescence recovery after photobleaching technique. Biochim Biophys Acta 943:367-370. CrossRef Medline

Naves LA, Van der Kloot W (2001) Repetitive nerve stimulation decreases the acetylcholine content of quanta at the frog neuromuscular junction. J Physiol 532:637-647. CrossRef Medline

Pearse BM (1976) Clathrin: a unique protein associated with intracellular transfer of membrane by coated vesicles. Proc Natl Acad Sci U S A 73: 1255-1259. CrossRef Medline

Perez-Gonzalez AP, Albrecht D, Blasi J, Llobet A (2008) Schwann cells modulate short-term plasticity of cholinergic autaptic synapses. J Physiol 586: 4675-4691. CrossRef Medline

Rizzoli SO (2014) Synaptic vesicle recycling: steps and principles. EMBO J 33:788-822. CrossRef Medline

Rizzoli SO, Betz WJ (2005) Synaptic vesicle pools. Nat Rev Neurosci 6:5769. CrossRef Medline

Rossano AJ, Chouhan AK, Macleod GT (2013) Genetically encoded pHindicators reveal activity-dependent cytosolic acidification of Drosophila motor nerve termini in vivo. J Physiol 591:1691-1706. CrossRef Medline

Royle SJ, Bright NA, Lagnado L (2005) Clathrin is required for the function of the mitotic spindle. Nature 434:1152-1157. CrossRef Medline

Saheki Y, De Camilli P (2012) Synaptic vesicle endocytosis. Cold Spring Harb Perspect Biol 4:a005645. CrossRef Medline

Sakaba T, Schneggenburger R, Neher E (2002) Estimation of quantal parameters at the calyx of Held synapse. Neurosci Res 44:343-356. CrossRef Medline

Sato K, Ernstrom GG, Watanabe S, Weimer RM, Chen CH, Sato M, Siddiqui A, Jorgensen EM, Grant BD (2009) Differential requirements for clathrin in receptor-mediated endocytosis and maintenance of synaptic vesicle pools. Proc Natl Acad Sci U S A 106:1139-1144. CrossRef Medline

Schikorski T, Stevens CF (1997) Quantitative ultrastructural analysis of hippocampal excitatory synapses. J Neurosci 17:5858-5867. Medline

Shupliakov O, Löw P, Grabs D, Gad H, Chen H, David C, Takei K, De Camilli P, Brodin L (1997) Synaptic vesicle endocytosis impaired by disruption of dynamin-SH3 domain interactions. Science 276:259-263. CrossRef Medline

Takamori S, Holt M, Stenius K, Lemke EA, Grønborg M, Riedel D, Urlaub H, Schenck S, Brügger B, Ringler P, Müller SA, Rammner B, Gräter F, Hub JS, De Groot BL, Mieskes G, Moriyama Y, Klingauf J, Grubmüller H, Heuser J, et al. (2006) Molecular anatomy of a trafficking organelle. Cell 127:831-846. CrossRef Medline

Willox AK, Royle SJ (2012) Stonin 2 is a major adaptor protein for clathrinmediated synaptic vesicle retrieval. Curr Biol 22:1435-1439. CrossRef Medline

Wu LG, Betz WJ (1998) Kinetics of synaptic depression and vesicle reycling after tetanic stimulation of frog motor nerve terminals. Biophys J 74: 3003-3009. CrossRef Medline

Wu LG, Ryan TA, Lagnado L (2007) Modes of vesicle retrieval at ribbon synapses, calyx-type synapses, and small central synapses. J Neurosci 27 : 11793-11802. CrossRef Medline

Zhang X, Bao L, Ma GQ (2010) Sorting of neuropeptides and neuropeptide receptors into secretory pathways. Prog Neurobiol 90:276-283. CrossRef Medline 